\title{
A Lagrangian analysis of a developing and non-developing disturbance observed during the PREDICT experiment
}

\author{
B. Rutherford and M. T. Montgomery \\ Naval Postgraduate School, Monterey, CA, USA \\ Correspondence to: B. Rutherford (bdruther@nps.edu) \\ Received: 1 September 2011 - Published in Atmos. Chem. Phys. Discuss.: 19 December 2011 \\ Revised: 10 August 2012 - Accepted: 14 November 2012 - Published: 3 December 2012
}

\begin{abstract}
The problem of tropical cyclone formation requires among other things an improved understanding of recirculating flow regions on sub-synoptic scales in a time evolving flow with typically sparse real-time data. This recirculation problem has previously been approached assuming as a first approximation both a layer-wise two-dimensional and nearly steady flow in a co-moving frame with the parent tropical wave or disturbance. This paper provides an introduction of Lagrangian techniques for locating flow boundaries that encompass regions of recirculation in timedependent flows that relax the steady flow approximation.

Lagrangian methods detect recirculating regions from time-dependent data and offer a more complete methodology than the approximate steady framework. The Lagrangian reference frame follows particle trajectories so that flow boundaries which constrain particle transport can be viewed in a frame-independent setting. Finite-time Lagrangian scalar field methods from dynamical systems theory offer a way to compute boundaries from grids of particles seeded in and near a disturbance.

The methods are applied to both a developing and nondeveloping disturbance observed during the recent predepression investigation of cloud systems in the tropics (PREDICT) experiment. The data for this analysis is derived from global forecast model output that assimilated the dropsonde observations as they were being collected by research aircraft. Since Lagrangian methods require trajectory integrations, we address some practical issues of using Lagrangian methods in the tropical cyclogenesis problem. Lagrangian diagnostics are used to evaluate the previously hypothesized import of dry air into ex-Gaston, which did not re-develop into a tropical cyclone, and the exclusion of dry air from pre-Karl, which did become a tropical cyclone and later a major hurricane.
\end{abstract}

\section{Introduction}

\subsection{The genesis problem and pouch hypothesis}

The prediction of tropical cyclogenesis ${ }^{1}$ is an important problem in tropical meteorology and geophysical fluid dynamics. While much effort has been devoted to the study of mature tropical cyclones, and their track and intensity estimates, only recently has a significant research effort been dedicated to the problem of the transformation of a tropical disturbance into a tropical depression.

A new paradigm for tropical cyclogenesis, dubbed the "marsupial paradigm", was recently developed by Dunkerton, Montgomery, and Wang (Dunkerton et al., 2009) (hereafter DMW09). This work, and that of Wang (2012) showed that a region of recirculation along a propagating Easterly wave occurred in all pre-genesis cases analyzed therein. The center of the sub-synoptic scale circulation, defined as the intersection of the parent wave's trough axis and the wave's critical latitude in the lower troposphere, was demonstrated to be the preferred location of cyclogenesis. The region of recirculation, coined the "pouch", protects the low- to midlevel embryonic vortex from adverse conditions, while the

\footnotetext{
${ }^{1}$ The glossary on NOAA's Hurricane Research Division's website uses tropical cyclone as the generic term for a nonfrontal synoptic-scale low-pressure system over tropical or sub-tropical waters with organized convection (i.e. thunderstorm activity) and a definite cyclonic surface wind circulation." Notably, this definition does not invoke any wind threshold. The same glossary defines a "tropical depression" as a tropical cyclone with maximum sustained surface winds of less than $17 \mathrm{~m} \mathrm{~s}^{-1}$ (34 kt, $39 \mathrm{mph}$ ) and, in the Atlantic and Eastern Pacific Basins, a "tropical storm" as a tropical cyclone with surface winds between $17 \mathrm{~m} \mathrm{~s}^{-1}$ and $33 \mathrm{~m} \mathrm{~s}^{-1}$. In this study we will define genesis as the formation of a tropical depression and we impose no formal threshold on wind speed.
} 
proto-vortex within is nurtured and amplified by convection within the pouch. The three main hypotheses of the marsupial paradigm can be summarized as follows:

1. The cat's eye region within the critical layer has cyclonic recirculation with little strain deformation, and its center serves as the focal point for vorticity aggregation of convectively generated cyclonic vertical vorticity and their vortical remnants.

2. Air inside the cat's eye is repeatedly moistened by convection and is protected to some extent from the lateral intrusion of dry air.

3. The parent wave is maintained and possibly enhanced by convectively amplified mesoscale eddies within the wave.

\subsection{Cases from the PREDICT experiment}

The marsupial paradigm was tested in part during the PreDepression Investigation of Cloud systems in the Tropics (PREDICT) experiment. The PREDICT experiment was designed to investigate the problem of genesis during the 2010 Atlantic hurricane season through a series of flights into and data analysis of developing and non-developing tropical disturbances. Along with flight-level and dropsonde data, observational satellite data was analyzed and an ensemble of numerical weather forecast models were run each day during the experiment. A description of the overall experiment, as well as a summary of some first results, is given by Montgomery et al. (2012).

In a follow-up paper, Smith and Montgomery (2012) carried out a more in-depth analysis of the convective environment in a subset of developing and non-developing tropical disturbances during the last four week period of the experiment. In all systems, there is a tendency for the lower troposphere to moisten, but in the system that did not develop, the middle and upper troposphere became progressively drier during the five missions. The most prominent difference between the non-developing system and the two systems that developed was the much larger reduction of equivalent potential temperature between the surface and a height of $3 \mathrm{~km}$, typically $25 \mathrm{~K}$ in the non-developing system, compared with only $17 \mathrm{~K}$ in the systems that developed. Conventional wisdom would suggest that, for this reason, the convective downdraughts would be stronger in the non-developing system and would thereby act to suppress the development. Smith and Montgomery (op. cit.) presented an alternative hypothesis that the drier mid-level air weakens the convective updraughts and thereby weakens the amplification of system relative vorticity necessary for development. Although detailed testing of the alternative hypothesis is forthcoming (Kilroy and Smith, 2012), the findings of Montgomery et al. (2012) and Smith and Montgomery (2012) raise the important question of what caused the progressive drying for the non-developing disturbance and what prevented drying in the developing disturbances? As argued in DMW09, the answer to this question requires the realization of the Lagrangian nature of the genesis problem and the importance of identifying intrinsic flow boundaries that limit/enable the transport and mixing of adiabatic invariants between the potential development region and its environment.

The role of structures and boundaries is important for understanding the transport of moisture and absolute vertical vorticity, two important elements in the genesis of a tropical cyclone (DMW09). Considering this problem in timedependent velocity fields could ultimately lead to better prediction of cyclogenesis.

The cases that are analysed in this study are the developing case of Karl and the nondeveloping case of Gaston. The overall evolution of the disturbances can be seen in Fig. 4, which shows the evolution of relative humidity, the Okubo-Weiss parameter, and vorticity averaged over a 3 degree radius circle surrounding the circulation center. Karl remained moist at all levels while Gaston started to dry at $700 \mathrm{hPa}$ and above. One of the outcomes of this work will be an explanation of the drying in Gaston and the moistening in the Karl disturbance due to the interaction of the disturbances with their environments through the inspection of flow boundaries.

The study of flow kinematics during genesis is important for validating both aspects of the second hypothesis of the marsupial paradigm for candidate disturbances. The second hypothesis requires Lagrangian recirculation along the travelling parent wave to protect the pouch and also requires that the lateral intrusion of dry air be minimal. Both of these criteria can be verified by finding persistent boundaries at the edge of the primary circulation which allow recirculation within the nascent vortex and limit interaction from the environment. Recirculation of fluid parcels in a time-dependent flow does not necessarily occur in regions of instantaneous positive cyclonic vorticity, but only occurs when the parcel remains entrained for a sufficiently long time.

The Okubo-Weiss (OW) criterion (as defined herein) and vorticity are Eulerian measures of rotation commonly used in atmospheric predictions and analysis. Vorticity is defined mathematically as the curl of the velocity field and represents twice the local rotation rate of a fluid particle. The (OW) criterion helps characterize vortex resilience by measuring the relative role of vorticity in relation to strain and shear, and helps reveal particles that have high vorticity and low strain deformation and remain entrained in vortices.

\subsection{Flow boundaries}

Flow boundaries in the Eulerian or Earth-relative frame of reference are seen by streamlines. These streamlines are commonly used by forecasters to declare a tropical depression when closed circulation is seen. However, it is well known that particle trajectories may cross Eulerian streamlines in time dependent flows. Developing disturbances can 
alternatively be viewed in a storm-relative frame of reference travelling at the propagation speed of the parent Easterly wave. We refer to this reference frame as the co-moving frame. In this co-moving frame, streamlines often show closed circulation in the wave trough at sufficiently large wave amplitude prior to the declaration of a tropical depression. The use of the co-moving frame has led to a clearer picture of the background flow geometry that favors subsequent development of a disturbance. However, this viewpoint is still not widely used in forecasting applications.

Streamlines in a co-moving reference frame formed flow boundaries for a steady tropical cyclone vortex studied by Riemer and Montgomery (2011), and were shown to influence the import of dry air to the inner-core of the vortex. Similar boundaries were found during the PREDICT experiment by Evans et al. (2011).

In the cases studied during PREDICT, the time-variation of the translation speed was sometimes sufficiently large that streamlines did not exhibit consistently closed circulation under small variations in the translation speed. In addition, the co-moving frame does not account for time-dependence of velocities, so the pouch boundary in the co-moving frame may not be impermeable to the transport of air parcels. The deficiencies of the co-moving frame can be resolved by considering the Lagrangian frame of reference following particle trajectories.

In contrast to the co-moving frame following a feature which assumes a feature-relative steady flow, the Lagrangian reference frame can be used to locate persistent flow boundaries in flows with time-dependence.

If the flow is steady, the stable and unstable manifolds of a hyperbolic fixed point, or saddle-point partition the flow. The manifolds are distinguished from other streamlines since they show maximal linear instability for particles located close to the manifolds.

The time-dependent analog of a hyperbolic fixed point is called a hyperbolic trajectory and is often associated with persistent though not necessarily fixed stagnation points of the steady flow. The hyperbolic trajectory may have stable and unstable manifolds. These finite-length manifolds are the material lines which show maximal linear stability/instability for finite times. Hyperbolic trajectories and manifolds were shown by Duan and Wiggins (1996) and Ide et al. (2002) to control particle transport and persist in time-dependent flows. The stable and unstable manifolds of a pair of hyperbolic fixed points were found by Rogerson (1999) to control transport in and out of a cat's eye region for a barotropic jet. In genesis cases, the time of hyperbolicity is short enough so that multiple intersections of manifolds does not generally occur.

Finite-time Lagrangian scalar-field methods were developed (Haller and Poje, 1997; Haller, 2001; Haller and Yuan, 2000; Pierrehumbert, 1991) as an efficient way of directly locating kinematic boundaries in time-dependent flows without computing a hyperbolic trajectory. These studies showed that the manifolds were the material lines that tended to have maximum times of linear instability. The manifolds can be located as maximal ridges in Lagrangian scalar fields, which measure finite-time separation directly from particle trajectories. These boundaries will hereafter be referred to as Lagrangian coherent structures (LCSs). LCSs are robust under time-variation of fluid velocities (Shadden, 2006), and velocity approximations (Haller, 2002), though the loss of hyperbolicity of the underlying saddle trajectory may alter the invariance of the LCS (Branicki and Wiggins, 2010). These methods detect LCSs, which form finite-time boundaries and govern the mixing and transport of tracers (Shadden et al., 2005; Haller and Yuan, 2000; Haller, 2000) and have proven useful in a variety of geophysical flows, see e.g. Salman et al. (2008); Tang et al. (2009); Koh and Legras (2002); Joseph and Legras (2002); Cohen and Schultz (2005).

Many powerful diagnostic tools have been developed by the dynamical systems community for use in time-dependent flows. However, these new methods are not well known to hurricane forecasters and tropical meteorologists.

For vortex dominated flows, time-scale finite-time Lagrangian methods were introduced. When the boundaries are difficult to see due to turbulence, statistical measures of trajectory motion give insight into the mixing and transport processes.

In this study, Lagrangian kinematic aspects of the pouch theory are explored by an analysis of trajectory motion and scalar fields derived from trajectory motion using methods from dynamical systems. LCSs are located as the maximal ridges of scalar fields. The stirring, which is modulated by the LCSs, shows the pathways for the entry of air into a candidate disturbance or pre-storm.

\subsection{Summary}

The outline of this paper is as follows. In Sect. 2, we provide an introduction to Lagrangian methods for time-dependent flows, and show how they can be made applicable to the flows considered during the PREDICT experiment. Section 3 provides numerical details of the Lagrangian computations as well as numerical details about the data obtained from the PREDICT experiment. Sections 4 and 5 present the primary results of this study. In Sect. 4, we consider the nature of time-dependence of fluid velocities during pre-genesis conditions and contrast Lagrangian boundaries to Eulerian streamlines. In Sect. 5, finite-time Lagrangian methods and Lagrangian statistical methods are applied to a developing and a non-developing disturbance. The LCSs are shown to be important for controlling the import of dry air into the core for a non-developing disturbance, and protecting the core of a developing disturbance. Conclusions and a future outlook are provided in Sect. 6. 


\section{Overview of mathematical methods}

The methods used in this study can be divided into measures of recirculation and the computation of kinematic boundaries. For both types of methods, we consider the behavior of particle trajectories. Let $\boldsymbol{x}_{0} \mapsto \phi_{t_{0}}^{t}\left(\boldsymbol{x}_{0}\right)$ be the flow map associated with the ordinary differential equation for fluid velocities

$\dot{\boldsymbol{x}}=\boldsymbol{u}(\boldsymbol{x}, t)$

governing the location of particle trajectories $\boldsymbol{x}\left(\boldsymbol{x}_{0}, t_{0}, t\right)$, that is, the solution of Eq. (1) with initial condition $\boldsymbol{x}\left(t_{0}\right)=\boldsymbol{x}_{0}$. The growth of perturbations to initial conditions can be approximated by the linearized velocity field,

$\dot{\xi}=\nabla \boldsymbol{u} \xi$

\subsection{OW}

Assuming a slowly varying flow, the growth of $\xi$ is determined by eigenvalues of $\nabla \boldsymbol{u}$. If $\boldsymbol{u}$ is divergence free, the eigenvalues of $\nabla \boldsymbol{u}$ are opposite. The tendency of rotation of an air parcel is determined by purely imaginary eigenvalues of $\nabla \boldsymbol{u}, \lambda_{ \pm}= \pm i \sqrt{\mathrm{OW}}$, when $\mathrm{OW}=\frac{1}{4}\left(\zeta^{2}-S_{n}^{2}-S_{s}^{2}\right)>0$. $S_{n}=u_{x}-v_{y}$ and $S_{s}=v_{x}+u_{y}$ are the normal and parallel strain rates, $\zeta=v_{x}-u_{y}$ is vorticity, and $i^{2}=-1$. In this sign convention, positive OW indicates that vorticity dominates strain. Negative OW values indicate regions of high strain, but particles in regions of negative OW do not generally experience persistent stretching even in a steady flow (Lapeyre et al., 1999). Hua and Klein (2002) provided a generalized OW criterion which incorporates time-dependent terms. An OW criterion incorporating divergent terms is studied by Lukovich and Sheperd (2005).

\subsubsection{Hyperbolic fixed point and manifolds}

Assume that a velocity field $\boldsymbol{u}(\boldsymbol{x}), \boldsymbol{x} \in \mathbb{R}^{2}$ is steady. A fixed point $\boldsymbol{x}_{h}$ is a point where velocities vanish, $\boldsymbol{u}\left(\boldsymbol{x}_{h}\right)=0$. We are concerned only with fixed points having hyperbolic or saddle-type stability. Hyperbolicity of $\boldsymbol{x}_{h}$ may be defined by $\nabla \boldsymbol{u}\left(\boldsymbol{x}_{h}\right)$ having eigenvalues of opposite signs, showing a direction of expansion and contraction. The stable manifold is the solution that converges to the hyperbolic fixed point along the eigendirection of the positive eigenvalue. The unstable manifold diverges from the hyperbolic fixed point in the eigendirection of the negative eigenvalue or converges backward in time. The stable and unstable manifolds are important solutions because they form a partition of the flow, which distinguish them from generic streamlines. Particles near the stable manifold depart and travel toward the unstable manifold once they approach the fixed point. The stable manifold shows maximal repulsion to nearby particles while the unstable manifold shows maximal attraction, (Haller and Yuan, 2000; Ottino, 1990).

\subsection{Objectivity of reference frames}

While the manifolds of a hyperbolic fixed point form a flow partition that is conceptually beneficial for describing the pouch boundary, the location of the fixed point and manifolds are dependent on the choice of translation speed. Moreover, the rotating flow present during cyclogenesis contributes a non-Galilean rotation to any fixed coordinate system. Thus flow boundaries in the co-moving frame may be significantly different than the flow boundaries that are associated with actual particle motion. An objective ${ }^{2}$ frame of reference following particle trajectories avoids many of the approximations employed previously in the observational and modeling studies testing the pouch theory, such as a nearly steady flow in a co-moving reference frame. In addition, objective measures do not require the location of a pouch center or the computation of the pouch translation speed.

\subsubsection{Time variation, hyperbolic trajectories, and lobe dynamics}

Analogs to a hyperbolic fixed point and its invariant manifolds may still exist in a time-dependent flow and have the same local kinematic properties as in a steady flow. A distinguished hyperbolic trajectory (DHT) is a particle trajectory that is the time-dependent analog of a hyperbolic fixed point and maintains linearized hyperbolic stability, with eigenvalues of opposite signs at each time. DHTs are often marked by persistent hyperbolic stagnation points at each fixed time, but the persistence of a hyperbolic fixed point is neither a sufficient or necessary condition for the existence of a hyperbolic trajectory. (Branicki and Wiggins, 2010). The stable and unstable manifolds of the DHT are the particle trajectories that approach the DHT forward/backward in time (Ide et al., 2002). The manifolds of the time-dependent flow are not crossed by trajectories, and form flow boundaries in the same manner that velocity streamlines do for steady flows (Malhotra and Wiggins, 1999; Coulliette and Wiggins, 2000). A key feature of manifolds in time-dependent flows is that the entire manifold evolves with time. Algorithms by Mancho et al. (2003) and Mancho et al. (2004) locate manifolds, but are sensitive to the persistence of stagnation points and timedependence of velocities. Algorithms by Koh and Plumb (2000) and Koh and Legras (2002) are not sensitive to the persistence of stagnation points. Similar to the steady flow case, the stable and unstable manifolds intersect at the hyperbolic trajectory.

\footnotetext{
${ }^{2}$ Objective coordinate transformations are of the form $\boldsymbol{x} \mapsto$ $\mathbf{Q}(t) \boldsymbol{x}+b(t)$, where $\mathbf{Q}(t)$ includes rotations of the coordinate system. These transformations are more general than Galilean transformations which are of the form $\boldsymbol{x} \mapsto \mathbf{Q} \boldsymbol{x}+c t$ where $\mathbf{Q}$ is a proper orthogonal matrix, which only includes translations. For a thorough overview of the definitions and choice of reference frame relating to vortices, see Haller (2005).
} 
Stable and unstable manifolds are allowed to intersect at points other than the DHT. If additional intersections occur, contained regions formed by the transverse intersections of the manifolds are called lobes. Lobe dynamics describes the transport of material contained within the lobes (Malhotra and Wiggins, 1999). If a hyperbolic trajectory is hyperbolic for a finite time interval, the manifolds are finite length.

\subsection{Finite-time Lagrangian methods}

In the absence of persistent stagnation points marking a DHT, finite-time Lagrangian methods may still locate manifolds. For a time-dependent flow, consider two nearby trajectories straddling a manifold. It is expected that these trajectories experience more relative separation than generic pairs of trajectories. Finite-time Lagrangian methods measure this separation and show LCSs which correspond to manifolds as the regions of maximal separation. Though the DHT is not computed directly, it can be inferred as the intersection of stable and unstable manifolds located near a persistent hyperbolic fixed point.

\subsubsection{Finite time Lyapunov exponents}

Small perturbations in the initial condition, $\boldsymbol{y}_{0}=\boldsymbol{x}_{0}+\boldsymbol{\xi}_{0}$, lead to a perturbed trajectory $\boldsymbol{y}(t)=\boldsymbol{x}(t)+\boldsymbol{\xi}(t)$. For sufficiently small $\left|\boldsymbol{\xi}_{0}\right|$, the perturbation $\boldsymbol{\xi}(t)$ can be approximated through the Jacobian of the flow map as

$\xi(t)=d_{x_{0}} \phi_{t_{0}}^{t}\left(\boldsymbol{x}_{0}\right) \boldsymbol{\xi}_{0}$,

which satisfies the variational equation

$\dot{\boldsymbol{\xi}}=\nabla \boldsymbol{u}(\boldsymbol{x}(t), t) \boldsymbol{\xi}$.

Consider a time range $\left[t_{0}, t_{0}+\delta t\right]$ with fixed integration time $\delta t$ which is finite but not necessarily small. The growth of $\xi(t)$ during at time $t+\delta t$ is approximated by

$\|\boldsymbol{\xi}(t)\|^{2}=\left\langle\boldsymbol{\xi}_{0},\left(d_{\boldsymbol{x}_{0}} \phi_{t_{0}}^{t_{0}+\delta t}\left(\boldsymbol{x}_{0}\right)\right)^{*}\left(d_{\boldsymbol{x}_{0}} \phi_{t_{0}}^{t_{0}+\delta t}\left(\boldsymbol{x}_{0}\right)\right) \boldsymbol{\xi}_{0}\right\rangle$.

where $*$ denotes a matrix transpose. The Cauchy-Green deformation tensor defined as

$$
\Delta\left(\boldsymbol{x}_{0}, t_{0}, t_{0}+\delta t\right)=\left(d_{\boldsymbol{x}_{0}} \phi_{t_{0}}^{t_{0}+\delta t}\left(\boldsymbol{x}_{0}\right)\right)^{*}\left(d_{\boldsymbol{x}_{0}} \phi_{t_{0}}^{t_{0}+\delta t}\left(\boldsymbol{x}_{0}\right)\right) .
$$

Maximal growth of the perturbation occurs when $\boldsymbol{\xi}(0)$ is aligned with the eigenvector corresponding to the larger eigenvalue, $\lambda_{\max }(\Delta)$, of $\Delta$,

$$
\begin{aligned}
\max _{\xi_{0}}\|\boldsymbol{\xi}(t)\| & =\sqrt{\left\langle\boldsymbol{\xi}_{0}, \lambda_{\max }\left(\Delta\left(\boldsymbol{x}_{0}, t_{0}, t_{0}+\delta t\right)\right) \boldsymbol{\xi}(0)\right\rangle} \\
& =\sqrt{\lambda_{\max }\left(\Delta\left(\boldsymbol{x}_{0}, t_{0}, t_{0}+\delta t\right)\right)}\left\|\boldsymbol{\xi}_{0}\right\| .
\end{aligned}
$$

The objective quantity

$$
\sigma\left(x_{0}, t_{0}, t_{0}+\delta t\right)=\frac{1}{2|\delta t|} \ln \lambda_{\max }\left(\Delta\left(x_{0}, t_{0}, t_{0}+\delta t\right)\right)
$$

is the FTLE (considered as a function of $\boldsymbol{x}_{0}$ ) at the initial time $t_{0}$.

\subsubsection{Lagrangian scalar fields}

Finite-time Lagrangian scalar-field methods compute a field of Lagrangian values on a grid of initial conditions. Lagrangian values are assigned the initial position $\boldsymbol{x}_{0}$. Note that for finite-times, the parameter $\delta t=t-t_{0}$ is the chosen integration time. The scalar field of FTLEs is computed by evaluating the FTLE on a grid of initial conditions. Repelling and attracting LCSs are maximal ridges of the forward $(\delta t>0)$ and backward $(\delta t<0)$ FTLE-fields, which play the role of stable and unstable manifolds over the finite integration time (Haller, 2000; Shadden et al., 2005; Shadden, 2006; Haller, 2002).

\section{Data from the PREDICT Experiment}

\subsection{Analysis data}

The data used for this study from the PREDICT experiment is taken from the ECMWF model, which outputs velocity and moisture data on an evenly spaced planar grid with a spatial resolution of 0.25 degrees and temporal output every $12 \mathrm{~h}$. Vertical levels for velocity data output are on constant pressure levels. In this study, the ECMWF model output and forecast data for the case of two tropical disturbances from 28 August to 17 September is used, during the times that Gaston and Karl were tracked. The disturbance that became Gaston had a visible pouch on 28 August, and was designated a tropical storm by National Hurricane Center forecasters on 30 August and was later downgraded on 2 September to an area of disturbed weather. The Ex-Gaston was trackable until 11 September, but never redeveloped. The disturbance that eventually became Karl had a well-defined surface low along the wave trough and associated pouch on 9 September, and lasted until 17 September. Further details on the evolution of these storms is given in Montgomery et al. (2012), while the Lagrangian behaviour of these storms is described in Sect. 4 .

The temporal output of forecast data is every $12 \mathrm{~h}$, with a $120 \mathrm{~h}$ forecast run each day. The use of Lagrangian methods to locate structures in the forecast data would reveal LCSs that would exist under the given forecast. The forecast data provides a way to compute repelling LCSs in real time, but will be addressed in a future study. For this study, we use only the available analysis output data at $0 \mathrm{~h}$ and $12 \mathrm{~h}$ each day.

This study focuses on the kinematic aspects of genesis, which when combined with the dynamic and thermodynamic aspects, see Smith and Montgomery (2012), will help provide a more complete picture of genesis in the real atmosphere. For the kinematic boundaries, we focus on the model analysis data which incorporates dropsonde data, but we do not use dropsonde data directly since the Lagrangian computations require trajectory integrations which are far simpler using the model output grids. 


\subsection{Trajectory computations}

All of the Lagrangian fields are computed daily in a 24 degree longitude by 18 degree latitude domain centered at the pouch center by seeding particles at 0.125 degree intervals and computing particle trajectories both forward and backward in time. The time interval for model data is $12 \mathrm{~h}$, but we use a 15 minute intermediate time step on a fourth order Runge-Kutta solver to better resolve trajectory curvature. Decreasing the intermediate time-step further showed no visible effects on Lagrangian fields. Due to the coarse temporal output and intrinsic curvature of the flow, we adopt a simple feature tracking scheme for the temporal interpolation of velocities at intermediate times. The scheme aligns particles at intermediate times to their feature-relative position at known model output times by advancing the entire feature at the mean-flow speed. For a particle $\boldsymbol{x}(t)$ and $t$ an intermediate time, the velocity of the particle at the known output time $t_{k}$ is $\boldsymbol{u}\left(\boldsymbol{x}\left(t_{k}\right)\right)=\boldsymbol{u}\left(\boldsymbol{x}(t)+c_{p}\left(t-t_{k}\right)\right)$ where $c_{p}$ is the computed translation speed. Linear interpolation in time is then used to obtain fluid velocities at the intermediate output times. Linear spatial interpolation is used to compute velocities after the feature alignment is done. This process improves on simple linear or bi-cubic interpolation since curved trajectories can be tracked through a translating vortex.

In order to balance the constraints of resolution, availability of information, and computational expense, we have chosen $48 \mathrm{~h}$ as the integration time. This time is long enough to resolve individual LCSs while maintaining coherence of LCS's across varying initial times. Shorter integration times did not resolve LCS's as well, while much longer integration times resolved LCSs but were computationally expensive and sometimes yielded spurious structures associated with the domain boundary. The occurrence of these spurious structures was reduced by allowing particles that contacted the southern domain boundary to travel zonally at the same speed as particles at the equator while further poleward motion is suppressed. Since they are based on particle trajectories, Lagrangian fields require $48 \mathrm{~h}$ of velocities, but only require the computed pouch location as a point of reference for visualization at the initial time of integration. As explained in Sect. 3.3, we assume that the flow is layerwise two-dimensional, and have chosen $500 \mathrm{hPa}, 600 \mathrm{hPa}$, $700 \mathrm{hPa}, 850 \mathrm{hPa}$, and $925 \mathrm{hPa}$ as vertical levels for all computations.

Vorticity and OW values require the computation of velocity gradients, which are computed through second-order finite differences.

\subsection{Simplifications used for this study}

During the PREDICT experiment, for each model output time, a pouch translation speed was computed to view the co-moving reference frame. Particle trajectories follow co-moving streamlines if the flow is steady in the co- moving frame and layer-wise two-dimensional. Finite-time Lagrangian methods require only the computation of particle trajectories, so the assumptions of a steady flow is unnecessary. Lagrangian structures are found independent of the co-moving frame of reference associated with a computed translation speed. However, the translation speed is still used to compute velocities at times between model output times as described above.

Lagrangian methods have been developed that are suitable for three-dimensional flows, (Haller, 2005, 2011; Rutherford and Dangelmayr, 2010). However, the interpretation of boundaries is easier also in two dimensions since the manifolds are one-dimensional. Justification for computing two-dimensional structures on horizontal layers in a threedimensional flow is provided by Rutherford et al. (2012), which showed that the two-dimensional projection of LCSs computed from three-dimensional trajectories were aligned across vertical levels up to $7 \mathrm{~km}$ in the vicinity of rotating deep convective structures; the flow geometry was nearly two-dimensional even though trajectory paths were not. A similar alignment of LCSs across vertical levels is shown in this study.

Despite the availability of 3-D Lagrangian methods, these methods can only be employed if 3-D trajectory locations are accurate. We use the simplification of a 2-D velocity field due to the higher reliability of 2-D motion from the available global model data. Trajectories from 2-D velocities are suitable for analysing large-scale motions associated with the primary pouch boundary since the tropical atmosphere is typically stratified except in convective regions (Holton, 2004), which typically occur inside the pouch boundary. A layerwise two-dimensional flow is further justified since convective features are not well resolved by global models, thus horizontal convergence/divergence is small. In convective regions, ascent times are on the order of $1 \mathrm{~h}$, which is much smaller than the gap between model output times. The horizontal width scale of strong convective features is approximately $10 \mathrm{~km}$ which is smaller than the highest global model output resolution of $25 \mathrm{~km}$. (Houze Jr. et al., 2009; Bell and Montgomery, 2010; Reasor, 2005)

Since we use a trajectory-based approach, the "vertical escape" of trajectories off of the horizontal slices is a concern of the simplification. While trajectories may escape, the persistence of coherent structures across vertical levels suggests that the flow geometry remains approximately planar even though trajectory motions may not. Approximate vertical alignment of LCSs is required for the LCSs to form a two-dimensional manifold in the three-dimensional flow.

\section{An analysis of time-dependence in tropical disturbances}

In this section, we examine the kinematic structures which form finite-time boundaries and control particle transport 
between the environment and the pouch. Examples of these boundaries will be shown for ex-Gaston while it was classified as a tropical disturbance. We find that while the Lagrangian boundaries are related to the Eulerian manifolds, time-dependence and finite-time hyperbolicity cause important differences.

\subsection{Reference frames}

In the co-moving frame, a clearer view of the geometry associated with recirculation can be seen by a saddle point often appearing outside of the pouch, and stable and unstable manifolds forming the pouch boundary, Fig. 1. The unstable manifold encloses the pouch, while the region between the stable and unstable manifolds is a pathway for environmental air to reach the circulation center. In the case that the stable and unstable manifolds of a single fixed point coincide, a teardrop shaped boundary is formed and blocks any transport into or out of the pouch. If the manifolds of a pair of fixed points coincide, a cat's eye boundary is formed. Figure $2 b$ shows the co-moving streamlines and a cat's eye boundary, along with trajectory locations which enter a circle of 3 degree radius around the pouch center (yellow) ${ }^{3}$ within $48 \mathrm{~h}$ at the same time that the streamlines are shown. Some particles that enter the pouch from the northeast not only cross Eulerian streamlines, Fig. 2a, but also cross the co-moving streamlines, Fig. 2b. Since the 3 degree circle is completely contained within the co-moving cat's eye, the trajectories shown do not represent all of the air that enters the pouch from outside the cat's eye. The co-moving frame does not account for all 2-D particle motion for two reasons. The first reason is that the co-moving frame is based on a translation speed that is assumed constant in both space and time. The second reason is that velocities in the co-moving frame are still time-dependent.

\subsection{Sensitivity to changes in translation speed}

Recirculation is the primary characteristic of a vortex which enables repeated moistening and vorticity amplification, and is generally not sensitive to small variations in the computed pouch translation speed. However, the flow boundaries outside the recirculation region directly impact which environmental air masses interact with the vortex and may be sensitive to variations in the translation speed. A slight change in translation speed may change the shape and location of manifolds. Time-variations and spatial variations between the inner-core circulation and the hyperbolic fixed point location of the pouch translation speed may alter the streamline

\footnotetext{
${ }^{3} \mathrm{~A}$ yellow circle in a figure represents a 3 degree radius circle about the pouch center, which was computed as the location of the intersection of the wave critical layer and wave trough axis. PGI38L and PGI44L refer to Gaston and Karl respectively. Longitude is given on a $0-360^{\circ}$ convention. In this convention, the longitude of St. Croix is $295.25^{\circ}$.
}



Fig. 1. Schematic of the hyperbolic fixed point and manifolds near a convergent vortex in a layer-wise two-dimensional flow.

structure in the co-moving frame. The time variation of the computed pouch translation speed over a 12 hour time interval may exceed $2 \mathrm{~m} \mathrm{~s}^{-1}$. Spatial variations in velocities between a pouch center and pouch boundary also often exceed $2 \mathrm{~m} \mathrm{~s}^{-1}$.

The effect of a spatially uniform $2 \mathrm{~m} \mathrm{~s}^{-1}$ change in translation speed to streamline locations is shown in Fig. 2c. There are saddle points to the northeast and west of the disturbance, and the northeast saddle point and associated streamlines constrains the movement of the particles to the north. The co-moving streamlines indicate that the particles to the north will move south toward the storm, but will be taken to the east as they move southward, and will not enter the storm. Streamlines computed with the different translation speed show a much larger pouch boundary and the manifolds of the western stagnation point do not form a complete enclosure around the pouch. The streamlines indicate that the particles from the north will now be recirculated within the pouch. In this example, the particle motion is more accurately described by the streamlines computed with the adjusted translation speed. In other cases, the particles' final locations are mistaken by the streamlines computed from either translation speed, where in fact, many particles outside the boundary enter the pouch. Since the particles originate from a region with low relative humidity, the streamlines underestimate the amount of dry air entering the pouch. The differences in translation speed tend to have less effect on the geometry of strong vortices (Riemer and Montgomery, 2011). Since the Lagrangian frame does not require a translation speed, it is not sensitive to variations in translation speed.

\subsection{LCSs}

LCSs are the finite-time approximations to stable and unstable manifolds of a hyperbolic trajectory. In Fig. 2d, the forward (red) and backward time (blue) FTLE ridges for exGaston are shown at $700 \mathrm{hPa}$. There is a prominent attracting LCS (blue) and repelling LCS (red) which are in nearly the same location as the unstable and stable manifolds of the 


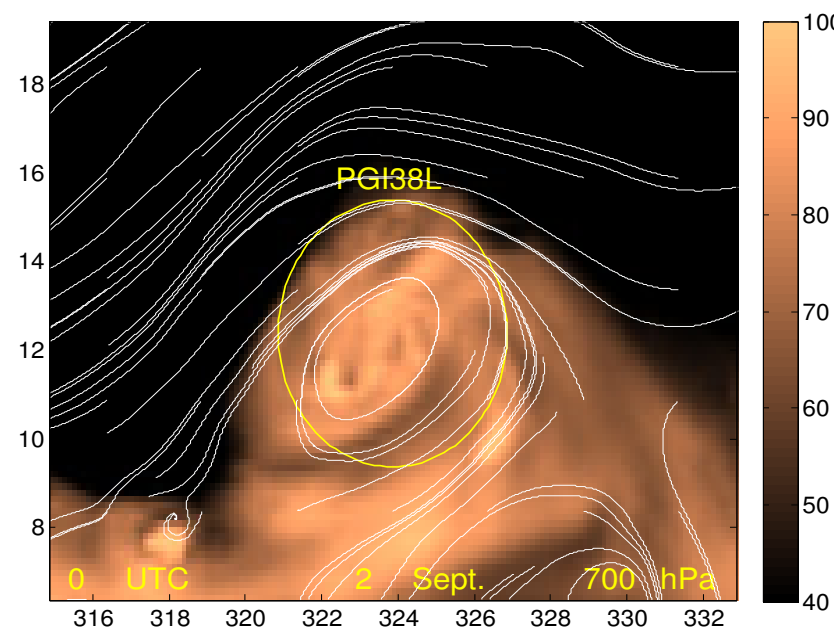

(a)



(c)

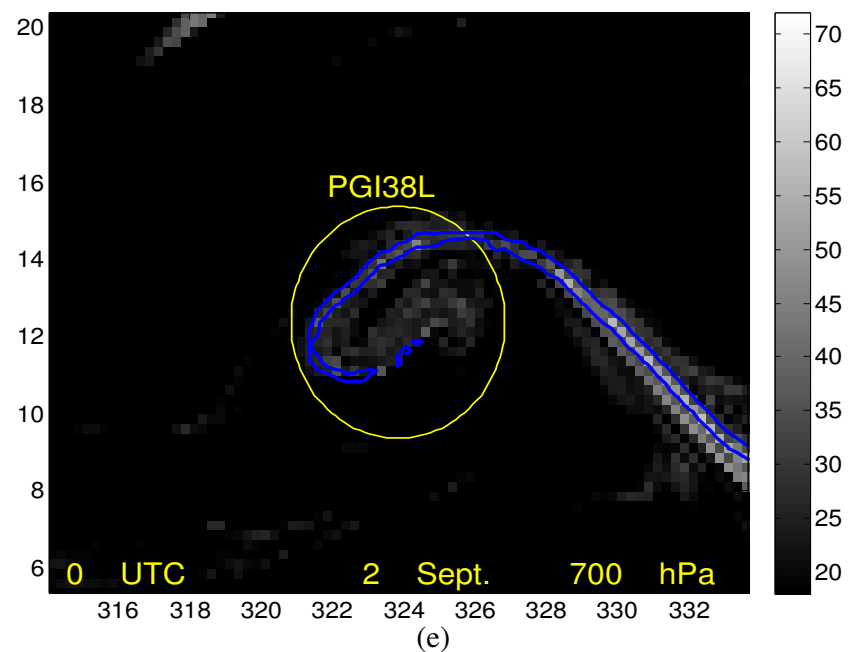

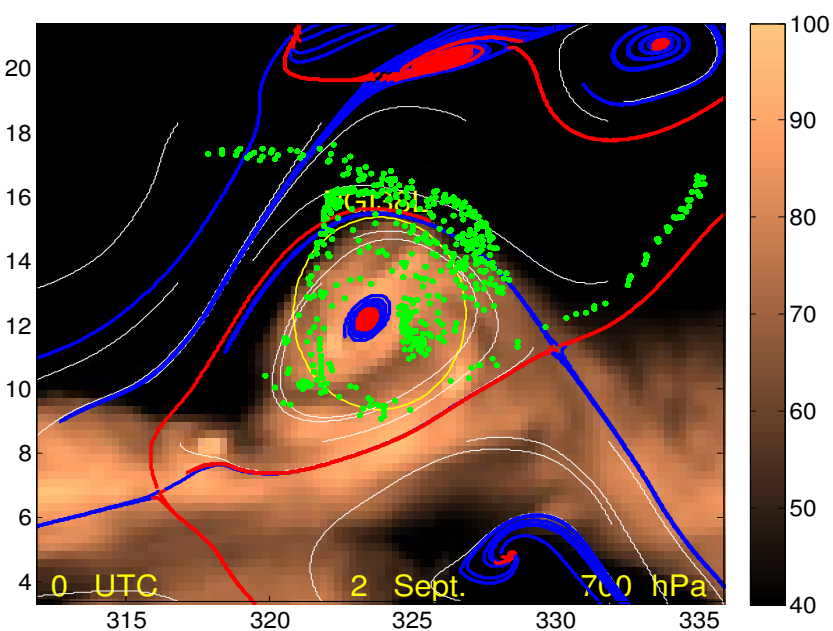

(b)

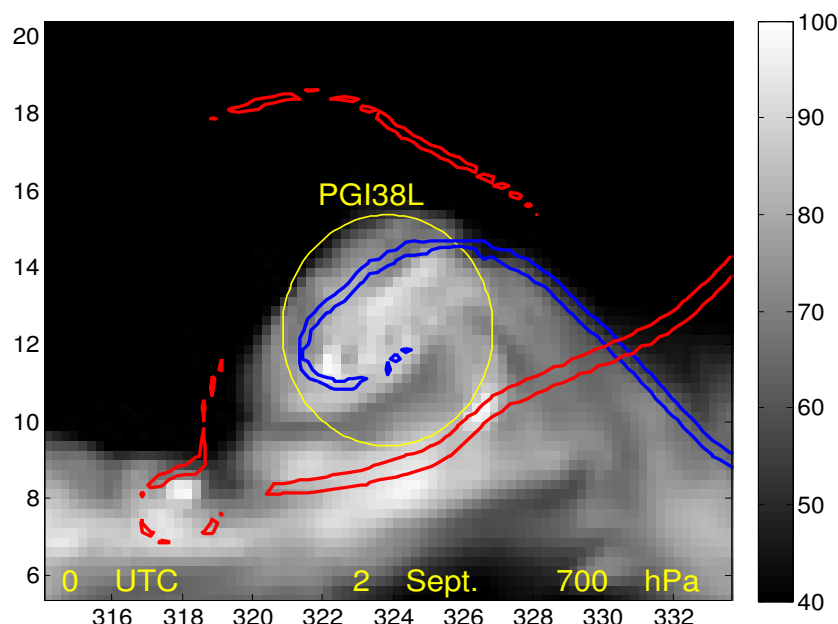

(d)

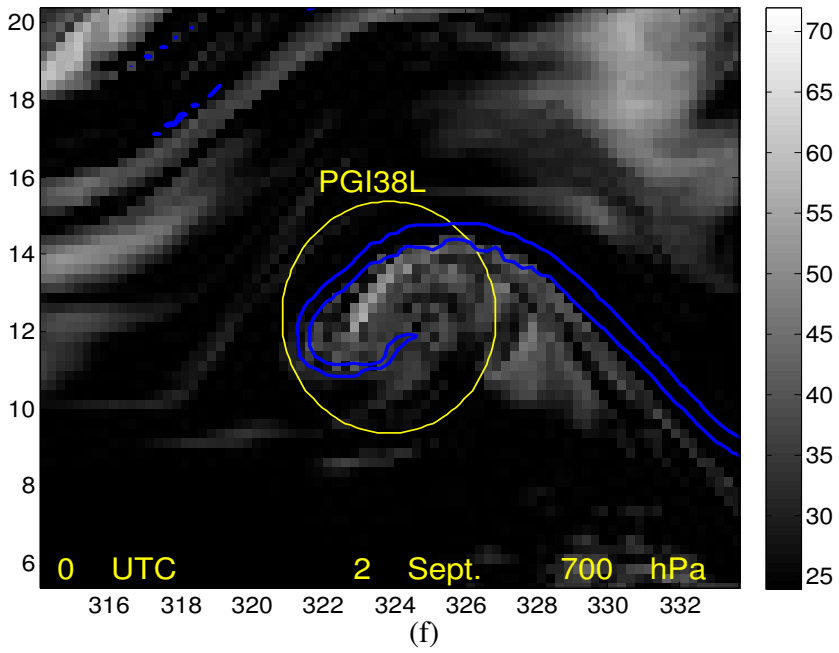

Fig. 2. Streamlines of Earth-relative velocities (a), streamlines of co-moving velocities (b), and streamlines of comoving velocities (c) with a $+2 \mathrm{~m} \mathrm{~s}^{-1}$ variation in translation speed shown on 2 September for ex-Gaston and overlaid on the relative humidity field (in \%) (a-c) Particle locations beginning in the pouch on 4 September are shown as green dots in (b). Co-moving stable and unstable manifolds are shown as red and blue lines, respectively, in (b) and (c). Attracting (blue) and repelling (red) LCSs are represented as level contours of the FTLE field at $80 \%$ of the maximal FTLE values, and are overlaid on the relative humidity field (d). The attracting LCSs are overlaid on the duration time of hyperbolic OW (hours) and duration time of elliptic OW (hours) in (e) and (f) respectively. 
eastern stagnation point of the co-moving frame. Unlike the streamlines, the LCSs have finite length, and do not connect with the western stagnation point. The LCSs are not crossed by particle trajectories. To reach the pouch center, trajectories travel around the LCS, which allows the set of trajectories from the northeast to enter the core. The LCS wraps around the core, allowing a recirculation region inside the LCS. In contrast to the eastern stagnation point, the manifolds of the western stagnation point are not represented by persistent LCSs. There are some very short LCS segments where the manifolds are located, but they do not connect with the manifolds of the eastern stagnation point to form an impenetrable boundary.

Differences in the LCSs and streamlines away from the hyperbolic fixed point are due to the time-variation in velocities. However, if the velocity near a stagnation point is slow, the co-moving manifolds may be computed while the LCS associated with the manifold does not exist, since there is not sufficient particle separation. The nonexistence of an LCS where a stable manifold resides provides one explanation to how particles appear to pass through the co-moving manifold.

FTLE values along repelling LCSs during periods of convergence are often smaller than the FTLE values along the attracting LCS. While LCSs with high FTLE values tend to be well resolved and persistent across varying initial time, repelling LCSs with smaller FTLE values do not persist across varying initial times. Small, isolated ridges with low FTLE values that do not persist across varying initial times are artifacts of the Eulerian flow that would disappear under a longer integration time, but are still visible due to finite integration times where the velocity at a particular output time still has some influence over trajectory locations.

The relation between the FTLE values and the time that particles are hyperbolic can be seen in Fig. 2e, where the attracting LCS is overlaid on the field showing the number of hours in a $72 \mathrm{~h}$ backward integration that particles satisfy the hyperbolic condition of the OW criterion. The strongest ridges are those where particles remain hyperbolic for the longest times, (Haller and Yuan, 2000). For this reason, we discard LCSs with low FTLE values since they tend to not be attracting or repelling to nearby particles for long periods of time, and would not be expected to have any meaningful influence as flow boundaries.

If attracting and repelling LCSs of the eastern and western hyperbolic trajectories, respectively, are resolved, and the LCSs have multiple intersections to the north of the pouch center near the moist and dry air boundary, transport across the Eulerian manifold is still possible through lobe dynamics. Enclosed lobes formed from intersecting segments of LCSs allow material to enter the circulation center through the deformation of the LCSs even when the Eulerian manifolds are persistent, though the air does not cross the LCSs. For the case of Gaston, lobe dynamics did not occur due to the finite hyperbolicity time and manifolds with short lengths that did not have multiple intersections. For Karl, lobe dynamics occurs well after genesis.

The foregoing considerations offer two explanations as to how the particles pass through the co-moving manifolds to bring dry air to the circulation center.

The relation of LCSs to physical quantities is shown in Fig. 3. Attracting LCSs define a sharp contrast between sets of particles based on the lengths of trajectory paths, Fig. 3a. In this case, the particles north of the LCS take much longer paths than those to the south of the LCS. Attracting LCSs are located along the gradients of physical quantities that are close to being materially conserved, such as ozone, Fig. 3b, and $\theta_{e}$, Fig. 3c. Particles with the highest potential vorticity and those satisfying the elliptic OW criterion tend to aggregate at the interior of the attracting LCS, Fig. 3d, e. In contrast to the Eulerian unstable manifold, the attracting LCS forms both the boundary that protects the vortex from lateral intrusion, and the interior of its curve marks the focal point of vorticity aggregation.

\section{Analysis of a non-developing and developing disturbance}

We now apply the Lagrangian methods to the cases of Gaston and Karl to show what additional insight these methods can provide in describing the development or non-development of a tropical depression.

\subsection{Evolution of ex-Gaston}

\subsubsection{Summary}

To provide insight as to why ex-Gaston did not re-develop, we look at the interaction of the storm with its environment at $700 \mathrm{hPa}$ and above which caused the pouch to become shallower despite a favourable environment at lower levels. A time series of pouch averaged metrics showing the evolution of Gaston is shown in Fig. 4. Gaston was declared a tropical storm on 1 September but was downgraded to a tropical depression on 2 September. On 1 September, Gaston resided in a relatively moist environment with high moisture at lower levels but surrounded by dry air at upper levels. The influence of the dry air can be seen by deep shear and pouch shear that is above $12 \mathrm{~m} \mathrm{~s}^{-1}$ prior to 1 September, and remains above $8 \mathrm{~m} \mathrm{~s}^{-1}$ on and after 1 September. Moisture values peaked on 1 September, and began to decline at $700 \mathrm{hPa}$ and above, as substantial dry air had penetrated the pouch at mid and upper levels, lowering the pouch-averaged relative humidity by approximately $20 \%$ by 5 September. Before 5 September, this intrusion was not noticeable at $700 \mathrm{hPa}$ in the relative humidity analysis data, and lower levels actually showed moistening (Montgomery et al., 2012). The interaction of dry air with the pouch suggests that the pouch was subjected to vertical wind shear (Davis and Ahijevych, 2011). Smith and Montgomery (2012) show that the dry air entering the pouch 




(a)

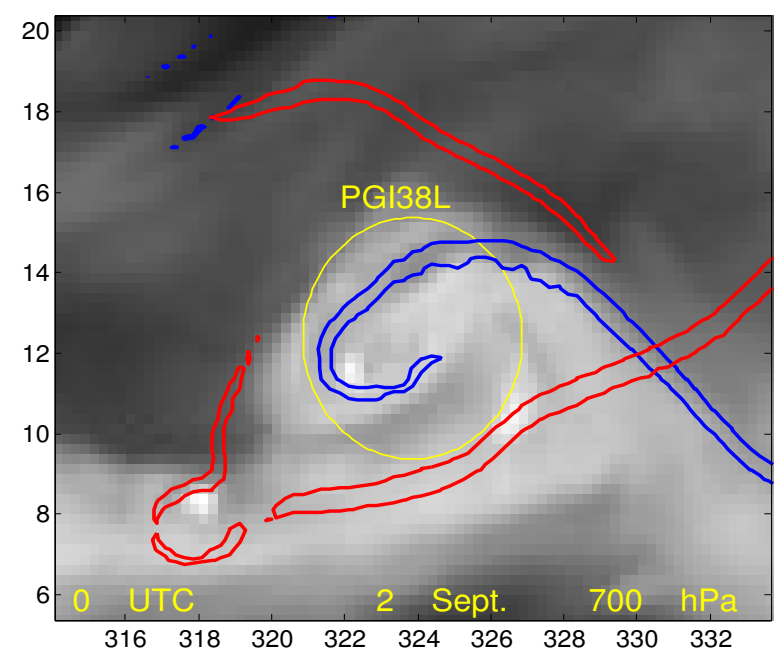

(c)

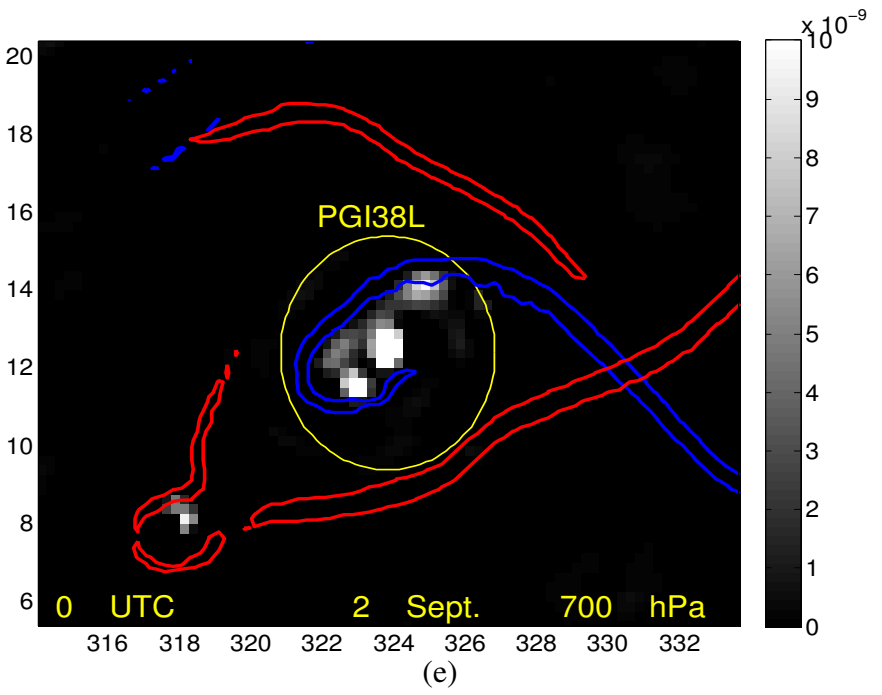

Fig. 3. The length of backward trajectory paths in degrees (a), $O_{3}$ mass fraction $\left(\mathrm{kg} \mathrm{kg}^{-1}\right)(\mathbf{b}), \theta_{e}(\mathrm{~K})(\mathbf{c})$, potential vorticity $\left(s^{-1}\right)(\mathbf{d})$, and OW $\left(\mathrm{s}^{-2}\right)(\mathbf{e})$ are shown for Gaston at 00:00 UTC on 2 September at $700 \mathrm{hPa}$ with attracting (blue) and repelling (red) LCSs are represented as level contours of the FTLE field at $80 \%$ of the maximal FTLE values. 
decreased $\theta_{e}$ in the mid to lower troposphere but did not produce widespread downdrafts strong enough to decrease the boundary layer $\theta_{e}$ on the pouch scale. On 1 September, the pouch has high relative humidity at all levels, but is surrounded by dry air. As the humidity decreased, the pouchaveraged OW and vorticity, Fig. 4c, e, also began to decline.

A time series of pouch-averaged relative humidity, vorticity and OW are shown in Fig. 4. Relative humidity values above $80 \%$ until 4 September, Fig. 4a, implies that dry air entering the pouch occurs at $700 \mathrm{hPa}$ and above and is compensated by convection and the associated vertical transport of moisture from below. This view is supported also by the study of the thermodynamics of ex-Gaston by Smith and Montgomery (2012).

The Lagrangian coherent structures and trajectory analysis will demonstrate that the intrusion of dry air was occurring laterally in the period from 1 to 5 September, but the air was presumably moistened from convection. However, the pouch structure was significantly degraded. 5 September marked the point where the intrusion of dry air and escape of moisture through ventilation could not be overcome by convective moistening from below. By this point, the pouch had already lost most of its vertical structure, as there was no upper level circulation remaining (Evans et al., 2011). Time series showing the evolution of forward and backward time Lagrangian quantities are shown in Fig. 5.

\subsubsection{Analysis of the flow field}

The co-moving manifolds are shown for Gaston from 1 to 6 September at $700 \mathrm{hPa}$ in Fig. 6. A persistent hyperbolic stagnation point can be seen to the east of the pouch at $700 \mathrm{hPa}$, though the manifolds do not completely protect the pouch center. On 2 September, an additional stagnation point appears to the west of the pouch, and a cat's eye configuration remains in place through 6 September, though it opens temporarily on 3 September.

The evolution of the Lagrangian fields at $700 \mathrm{hPa}$ is shown for 1 September through 6 September in Fig. 7, where attracting LCSs (blue) and repelling LCSs (red) are overlaid on the relative humidity field. There are LCSs present that are related to the co-moving manifolds of the eastern hyperbolic stagnation points. These structures are visible on 1 September, Fig. 7a, where an attracting LCS, shown in blue, is located in a similar location as the unstable manifold (red) of the eastern stagnation point of the cat's eye. This LCS has a tail southeast of the pouch and wraps cyclonically around the north side of the pouch. and forms the northern boundary. Near the location of the eastern stagnation point, a repelling LCS intersects the attracting LCS, and the intersection marks a hyperbolic trajectory that is persistent until 5 September when the manifolds no longer intersect. A repelling LCS is persistent and splits the large region of high moisture on the south side of the pouch, eliminating a large portion of that moisture from contributing to development through importing moist air.

A notable difference between the LCSs and the Eulerian manifolds is that the LCSs are of finite length because of the finite-time hyperbolicity of the hyperbolic trajectory. Since they are of finite length, they do not form a complete enclosure around the pouch of ex-Gaston.

A repelling LCS related to the western stagnation point does not intersect the attracting LCS at the northern edge of the pouch to form a cat's eye like boundary from air masses to the north, Fig. 7a-f. Since this boundary is not closed and the repelling LCS is not persistent, the attracting LCS controls the interaction of the pouch with dry air to the north. The attracting LCS forms most of the boundary to the north, and resides approximately 3 degrees from the pouch center. The attracting LCS forces air that is east of the structure to wrap around the pouch, and the air does not enter for at least two days.

Vertical wind shear pushes particles from a broad region of low relative humidity from the north toward the pouch (Davis and Ahijevych, 2011), and forces the LCS southward. This dry air does not directly enter the pouch as it is blocked by the attracting LCS, which forces the dry air mass to wrap around the west side of the pouch before it is entrained. The orientation of the repelling LCS indicates that the moist air to the south of the pouch will not be drawn inward.

By 2 September, the attracting LCS splits east of the pouch center, begins to effectively "unzip", Fig. 7d, and part of the LCS begins to travel cyclonically around the center. This branch rejoins the stationary branch on 4 September after making one revolution around the pouch, Fig. $7 d$, further constricting the moisture source from the ITCZ, Fig. 7d. The western repelling LCS rotates with the cyclonic flow toward the pouch center, and does not prohibit dry air from entering since it is not constrained by multiple intersection points. As it enters the pouch center, it disappears, and is replaced by a new repelling LCS to the north of the pouch center on 2 September, Fig. 7c. The replacement of the repelling LCS allows a region of dry air between the repelling LCS branches to cross where the co-moving streamline resides from 2 September to 4 September.

A pathway for material exiting the pouch also emerges due to a change in the flow field and is marked by the presence of a repelling LCS, which is entrained into the pouch center at $700 \mathrm{hPa}$ and above on 4 September. At the same time, the attracting LCS no longer encircles the pouch center. The aggregate contribution of the repelling LCS can be seen by the time series of forward time FTLE values in the Figure 5, which shows higher FTLE values at 500 and $700 \mathrm{hPa}$ beginning on 2 September at the time where significant mixing was occurring in the pouch. Repelling LCSs at the pouch center normally have smaller FTLE values but this LCS has FTLE values similar to those of attracting LCSs, indicating a shift from convergence to divergence within the pouch. After the repelling LCS appears and the ITCZ moisture source is 

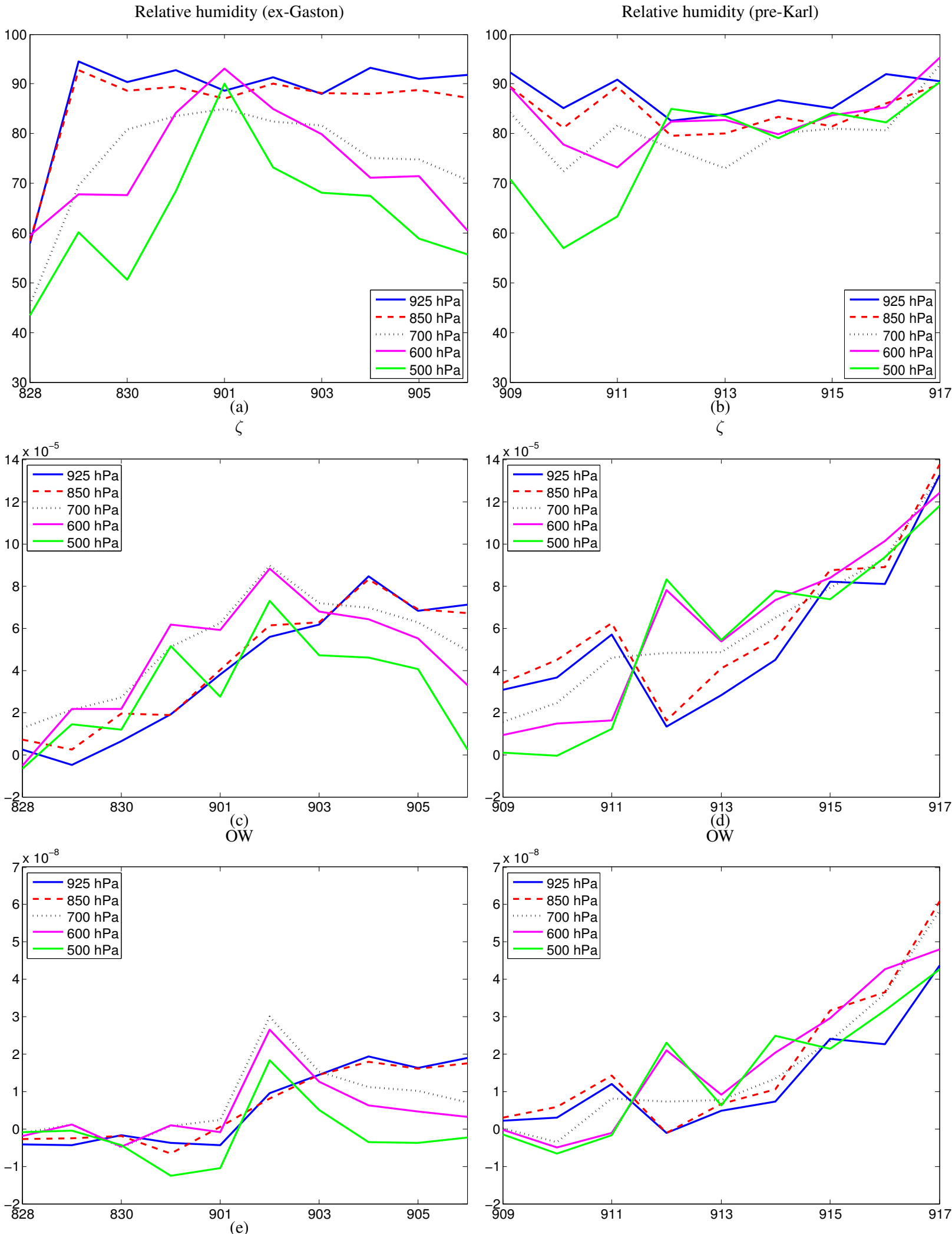

Fig. 4. Pouch averages of Eulerian quantities for the 2 degree circle surrounding the pouch are shown for ex-Gaston (left) and pre-Karl (right) for the relative humidity $(\%)(\mathbf{a}, \mathbf{b}), \zeta\left(s^{-1}\right)(\mathbf{c}, \mathbf{d})$, and Okubo-Weiss parameter $\left(\mathrm{s}^{-2}\right)(\mathbf{e}, \mathbf{f})$. The different curves represent vertical levels of $500 \mathrm{hPa}, 600 \mathrm{hPa}, 700 \mathrm{hPa}, 850 \mathrm{hPa}$, and $925 \mathrm{hPa}$. 


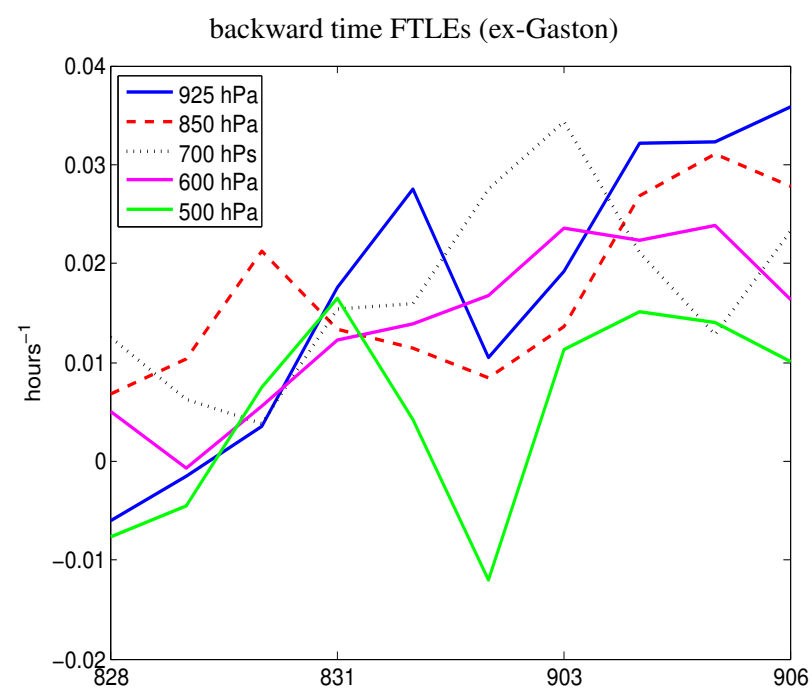

(a)

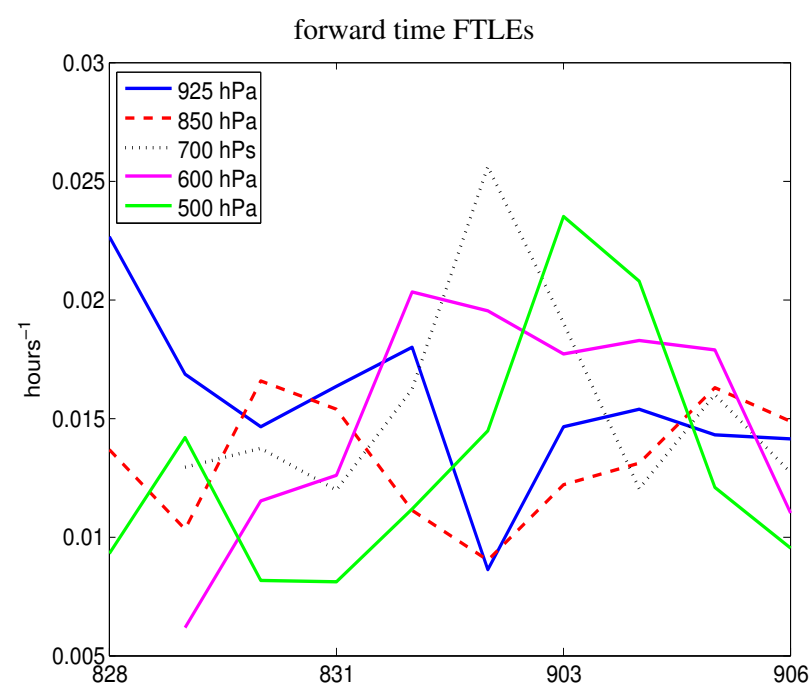

(c)

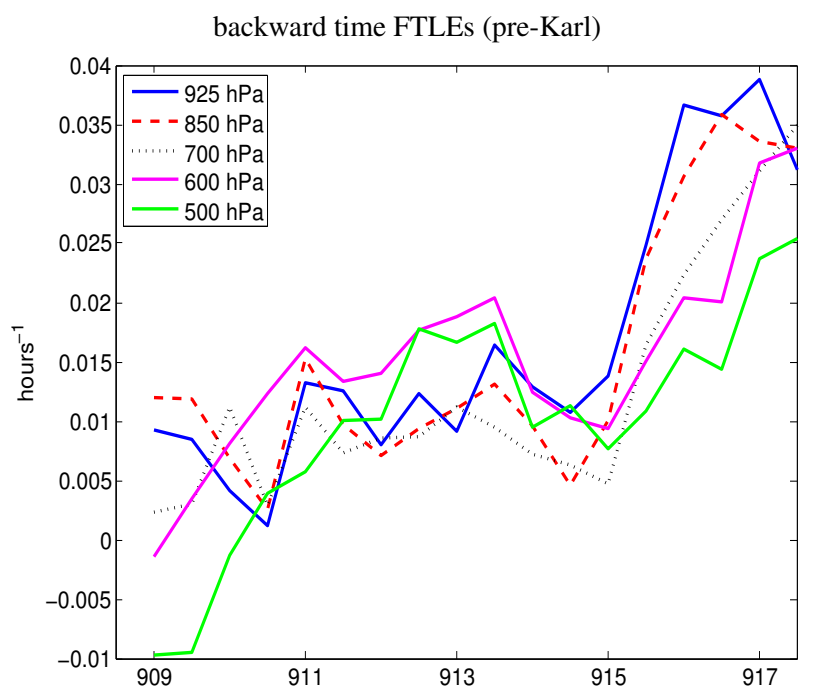

(b)

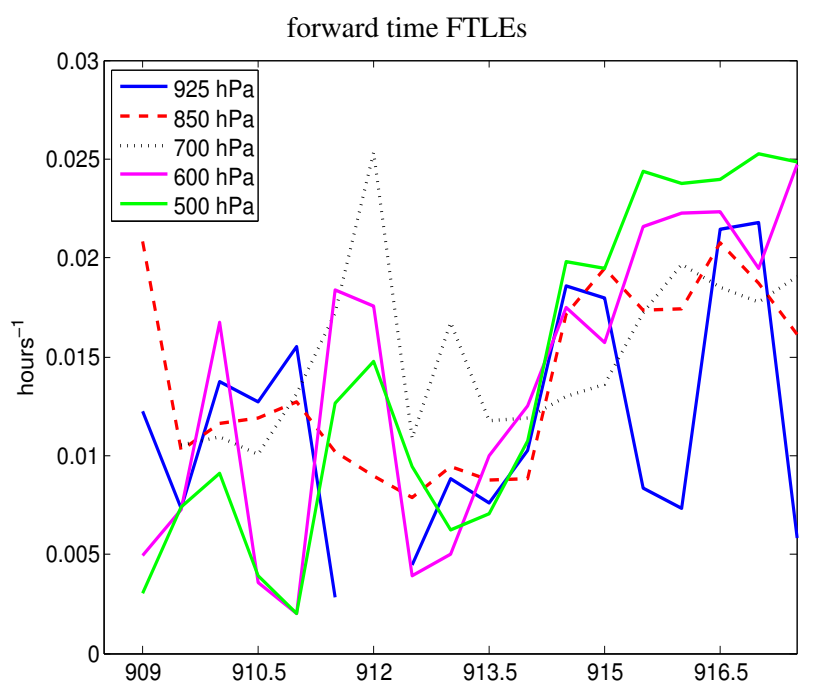

(d)

Fig. 5. Pouch averages of backward-time $(\mathbf{a}, \mathbf{b})$ and forward time $(\mathbf{c}, \mathbf{d})$ FTLE fields for a 2 degree circle surrounding the pouch are shown for Gaston (a, c) and Karl (b, d). The different curves represent vertical levels of $500 \mathrm{hPa}, 600 \mathrm{hPa}, 700 \mathrm{hPa}, 850 \mathrm{hPa}$, and $925 \mathrm{hPa}$.

removed, air from the pouch is allowed to leave the pouch to the west.

\section{Trajectory analysis}

The relation between LCSs and particle trajectories can be seen further by comparing the Lagrangian fields to a tracer field. Though it is not materially conserved, relative humidity may be used as a tracer field (by advecting the field forward in time and pretending there are no sources or sinks). The relative humidity tracer field, at $700 \mathrm{hPa}$ in Fig. 8 and $500 \mathrm{hPa}$ in Fig. 9, shows the initial moisture content of air parcels entering the pouch and confirms that some of the air entering the pouch is initially dry while the air exiting the pouch is initially moist. Attracting and repelling LCSs are overlaid in blue and red respectively. The relative humidity tracer field indicates that dry air is already entering laterally, Fig. 8a. From 2 September to 4 September, moist air persists at the circulation center, but some dry air passes to the center through the open pathway. By 3 September, the relative humidity tracer field, showing relative humidity values on 1 September, shows an intrusion of dry air in the pouch which entered from the southwest along the second repelling LCS branch. The lateral dry air intrusion is confirmed in the analyzed ozone field by a broad intrusion on the west side of the pouch, Fig. 8c, d. By 4 September, the air from the north has forced the attracting LCS southward and deformed the portion of the repelling LCS wrapping around the west side of the pouch. The net result is that the dry air intrusion from the 
north can enter the pouch directly without wrapping around from the west.

There are strong similarities between the tracer field and the attracting structures shown in Fig. 8a, b. In contrast, the relative humidity analysis data shown in Fig. 7 does not provide much information about the transport of particles. A similar scenario can be seen for pre-Karl in Figs. 11 and 13. The intrusion of dry air continues over the next 3 days, and by 5 September, the relative humidity tracer field shows that initially dry air has entered the pouch while moist air has exited the pouch to the west. The expulsion of moist air seen in the relative humidity tracer field occurred at the same time that the repelling LCS emerged at the pouch center. This repelling LCS and the absence of an attracting LCS provides a mechanism for moist air to be expelled from the center to the west of the storm.

The RH tracer field and LCSs can also be compared with the initial locations of particles that will enter the pouch in a two day period. These particle locations are computed as the final location of backward trajectories seeded inside a 3 degree radius circle surrounding the pouch center from 2 days in the future. The backward trajectories for ex-Gaston are shown in Fig. 6 for $700 \mathrm{hPa}$ from 1 to 6 September. Over this time period, there are many trajectories from the north and northeast of the pouch that enter the circulation. Comparing these locations to the $\mathrm{RH}$ tracer field indicates that many of the trajectories with initially low relative humidity values reach the pouch center.

The length of trajectory paths during a $72 \mathrm{~h}$ integration time are shown in Fig. 8e, f. The gradient of the curve lengths aligns with the attracting LCS. Though the initial positions of trajectories on opposite sides of the LCS may be very close, the length of their paths is very different. The moist air from the south takes a very short path to the circulation center while the dry air to the north takes a very long path, with average particle speeds of approximately $12 \mathrm{~m} \mathrm{~s}^{-1}$ for a $72 \mathrm{~h}$ integration time.

\section{Vertical structure}

The vertical structure of Gaston is shown on 1 and 2 September in Fig. 10. The low-level attracting LCSs (cyan) are nearly orthogonal to the LCSs at $700 \mathrm{hPa}$ and above (blue), showing that trajectory paths between vertical levels can be almost orthogonal even when the Eulerian boundaries are aligned. After 1 September, the attracting LCS is rotated and tilted, disrupting the vertical alignment of the pouch as the pouch boundary at $700 \mathrm{hPa}$ and above is south of the boundary below $700 \mathrm{hPa}$. The tilt between $500 \mathrm{hPa}$ and $700 \mathrm{hPa}$ can be seen by the lack of alignment of the eastern hyperbolic trajectory, Figs. $7 \mathrm{c}$ and $8 \mathrm{~d}$. A tilted pouch makes it possible for dry air which has come from the north to enter the low level circulation from aloft. However, the two-dimensional trajectory analysis as well as the thermodynamic analysis of Smith and Montgomery (2012) suggest that the dry air is from the north. On 2 September the already shallow pouch deteriorates further as the repelling LCS at $700 \mathrm{hPa}$ near the stagnation point detaches from the LCSs below $700 \mathrm{hPa}$, and the attracting LCS at $700 \mathrm{hPa}$ detaches from the LCSs below $700 \mathrm{hPa}$. The attracting LCSs at $700 \mathrm{hPa}$ actually align orthogonal to those below $700 \mathrm{hPa}$. The repelling LCS on the west side of the pouch has also become stronger at this time and intersects the attracting LCS near the pouch center.

Further change in the vertical alignment on the pouch occurs from 1 to 5 September. While the pouch boundary remains in the same location relative to the pouch center below $700 \mathrm{hPa}$, the boundary moves southward at $700 \mathrm{hPa}$ as particles from the north push the boundary southward. As the LCS is pushed southward, the circulation is tilted, Fig. 7.

In Fig. 10, the FTLE ridges are overlaid on relative humidity fields for ex-Gaston and pre-Karl at levels of $500 \mathrm{hPa}$, $700 \mathrm{hPa}$, and $925 \mathrm{hPa}$. The pouch boundary of ex-Gaston is displaced and rotated between vertical levels, while pre-Karl has far greater vertical coherence. For both storms, the manifolds associated with the saddle to the west of the pouch do not coincide with LCSs at multiple vertical levels.

Based on the foregoing results, the LCSs are related to the import of dry air, the vertical tilting of the vortex, a constriction of the ITCZ moisture source, and the expulsion of moist air when a repelling LCS is present at the center. The repelling LCS is particularly important since it is the signature of a vortex which is turned inside-out, shifting from a period of net inflow to net outflow. The LCS view differs from the study of Schubert et al. (1999), which showed a vorticity profile that was radially inverted due to shear instabilities. Because the radial profile of vorticity is approximately monotonic, the profile does not support these instabilities. The thermodynamic implications of a dying vortex are important also, as the planar outflow limits the ability of convection to transport moisture upward. For a developing vortex, the amplification of vorticity occurs ultimately through vortex tube stretching associated with convection. However, the presence of a repelling LCS wrapping around the vortex core indicates that convective activity is diminished since the air which has been transported inward is now mixed or transported outward. In Rutherford et al. (2012), the presence of a repelling LCS marks the end of the convective lifecycle of a three-dimensional vortical hot tower. The splitting of attracting LCSs that enclose vortices has also been seen in dying vortices (O'Farrell and Dabiri, 2010).

\subsection{Evolution of Karl}

\subsubsection{Summary}

Karl was classified as a tropical disturbance on 8 September, as an African Easterly wave merged with a surface trough to the north of South America. From 10 to 14 September, the near-surface circulation gradually increased in association with an approximate diurnal cycle of convective activity 

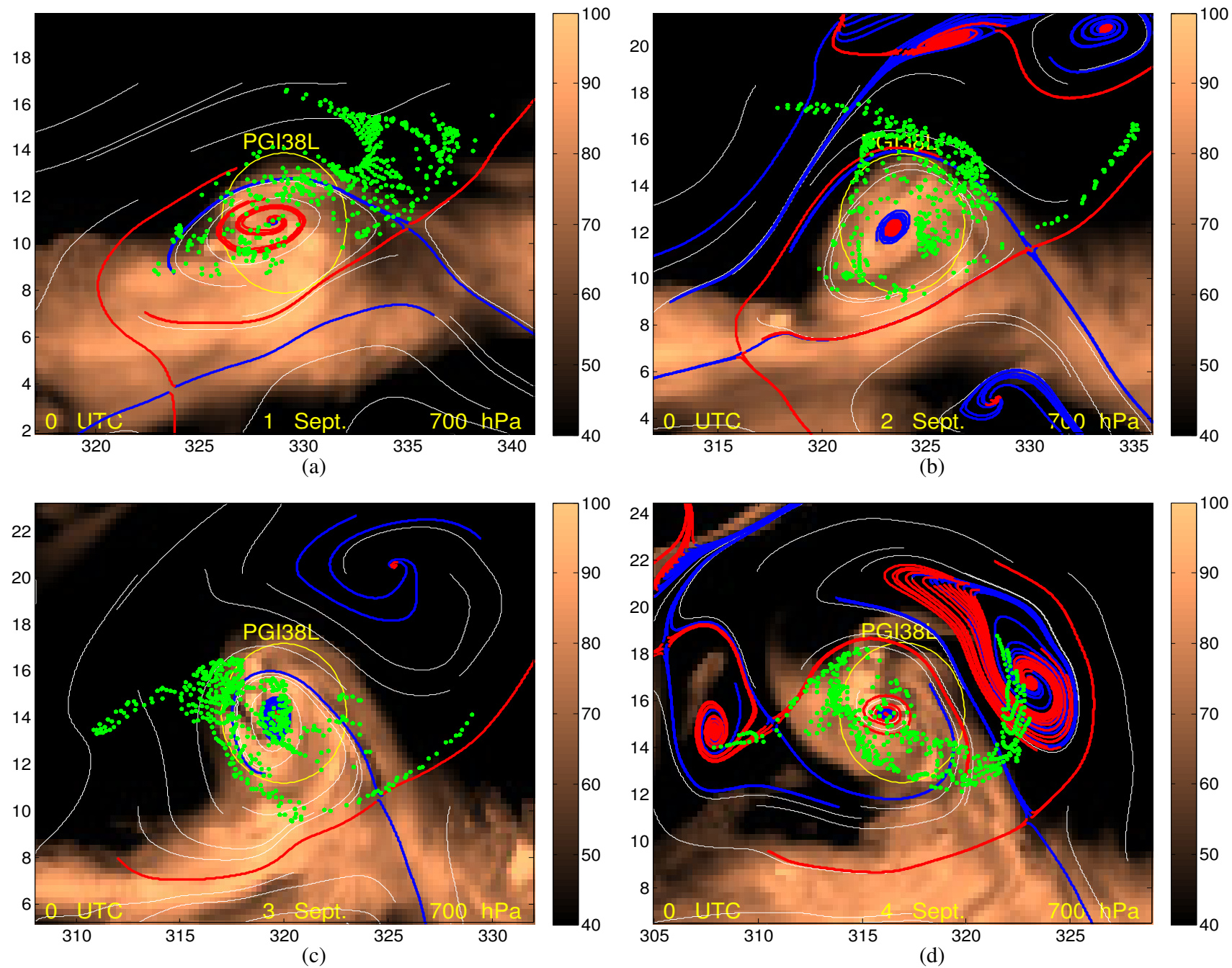

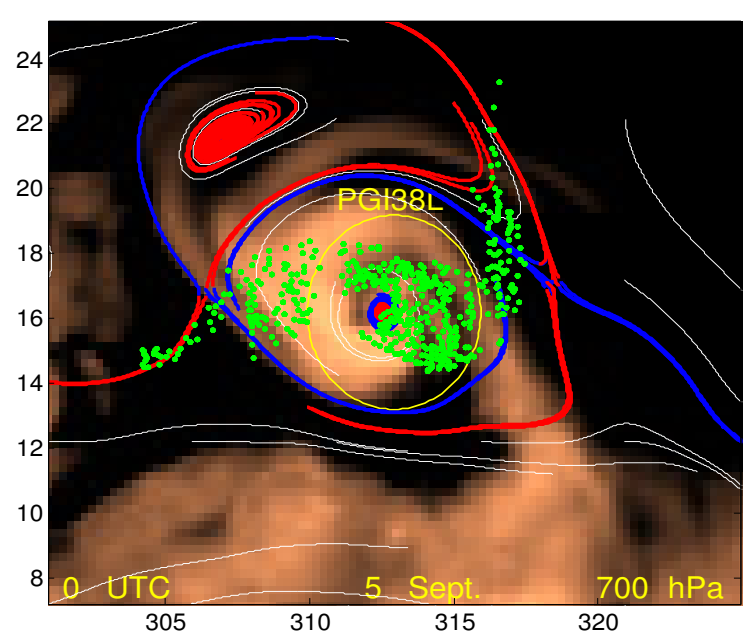

(e)



$-60$

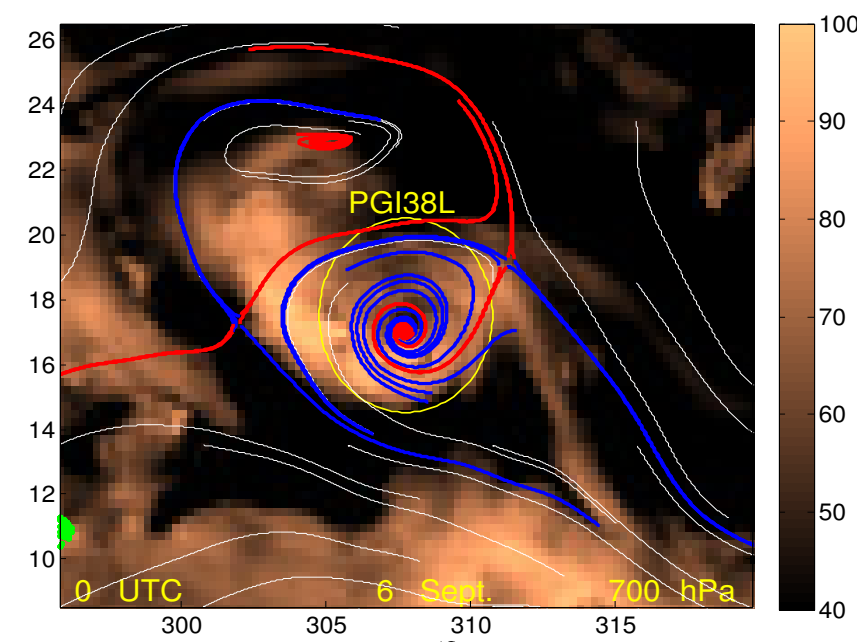

(f)

Fig. 6. The stable (red) and unstable (blue) manifolds of hyperbolic stagnation points and streamlines (white) of the co-moving frame are overlaid on the relative humidity field (\%) for Gaston from 1 September to 6 September at $700 \mathrm{hPa}$. Green dots indicate particles that will be within 3 degrees of the pouch center in $48 \mathrm{~h}$. 

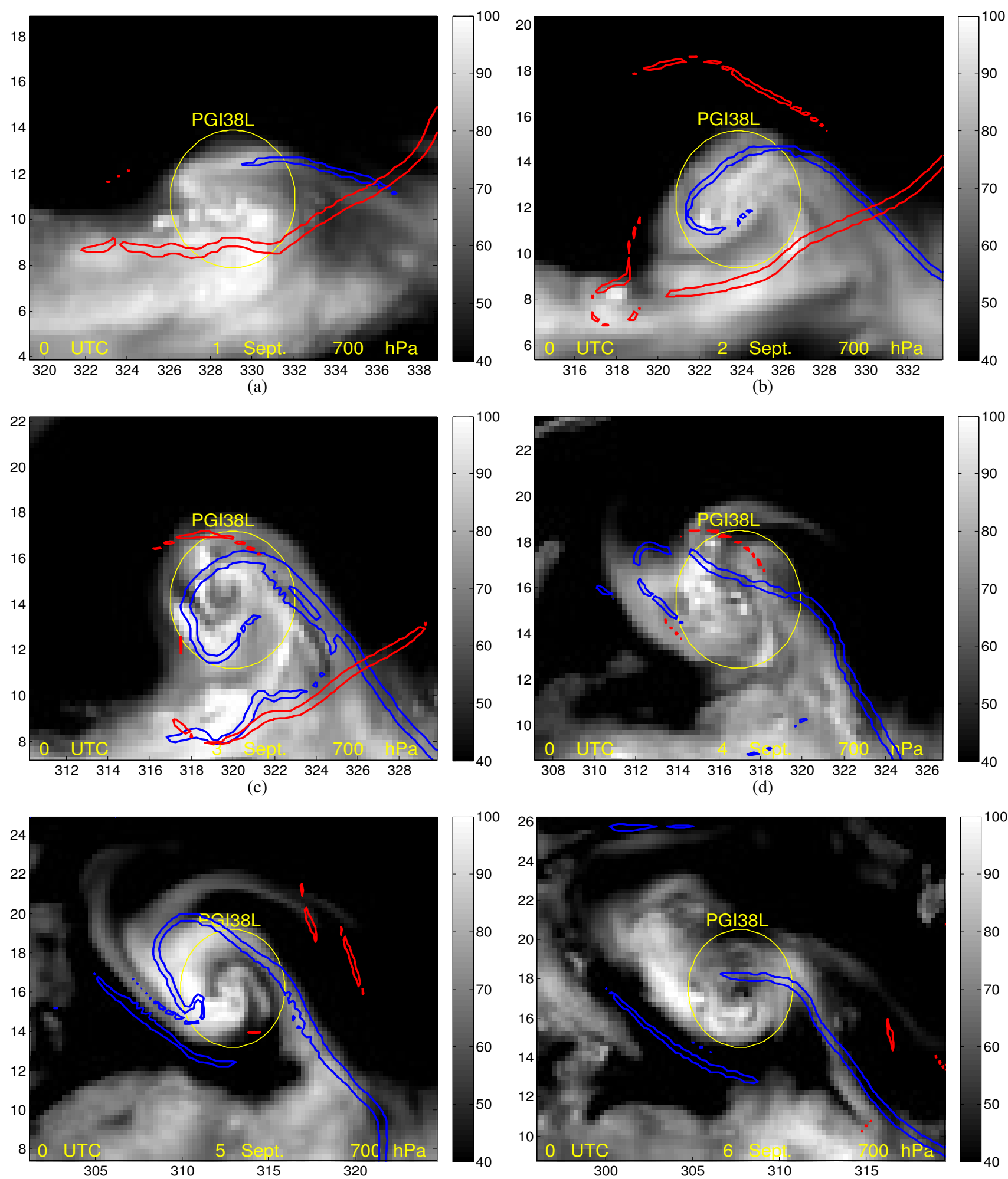

(e)

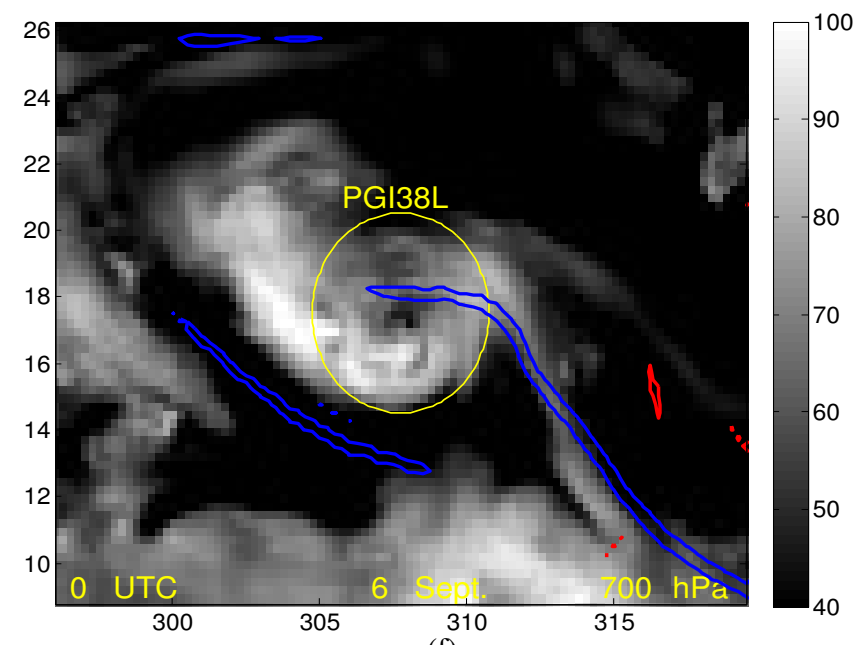

(f)

Fig. 7. Attracting LCSs (blue) and repelling LCSs (red) are represented as level contours of the FTLE field at $80 \%$ of the maximal FTLE values and overlaid on the relative humidity field ( $\%$ ) at $700 \mathrm{hPa}$ for ex-Gaston from 1 to 6 September. 


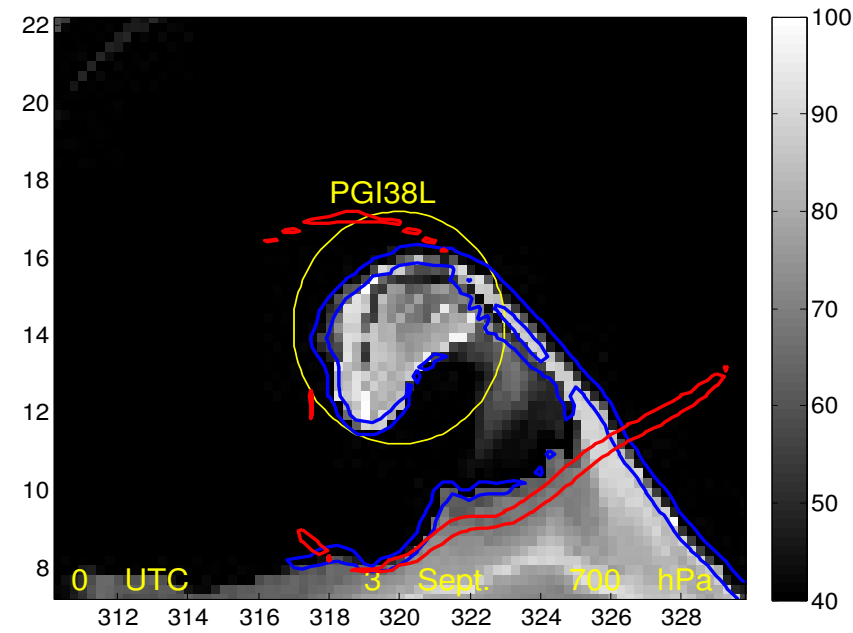

(a)



(c)

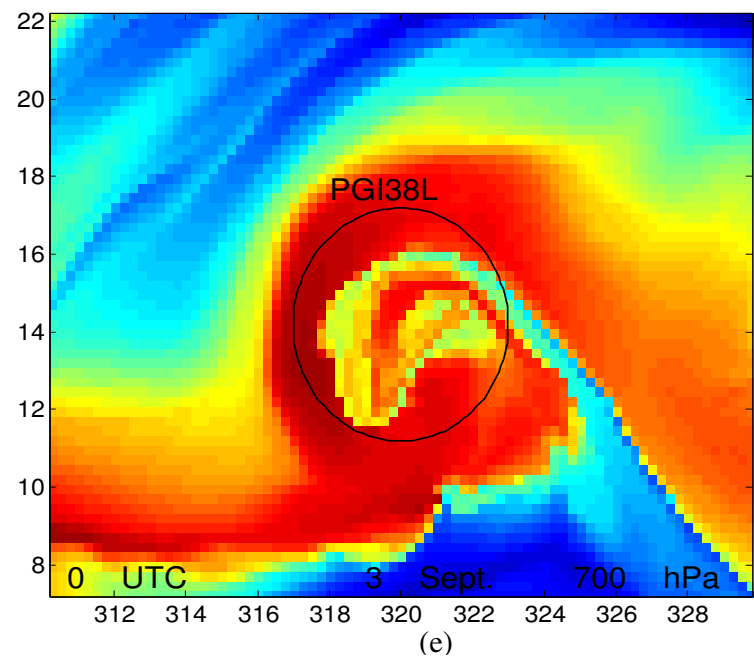

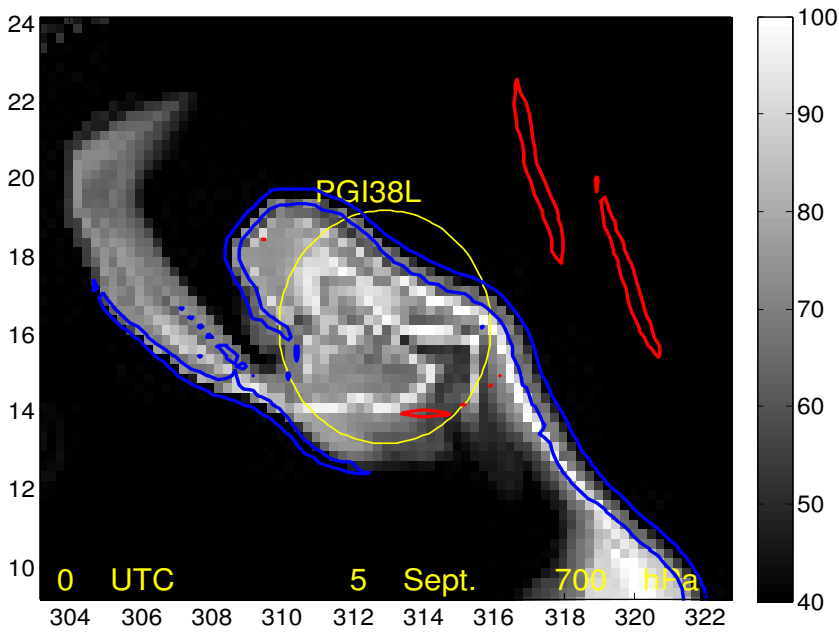

(b)

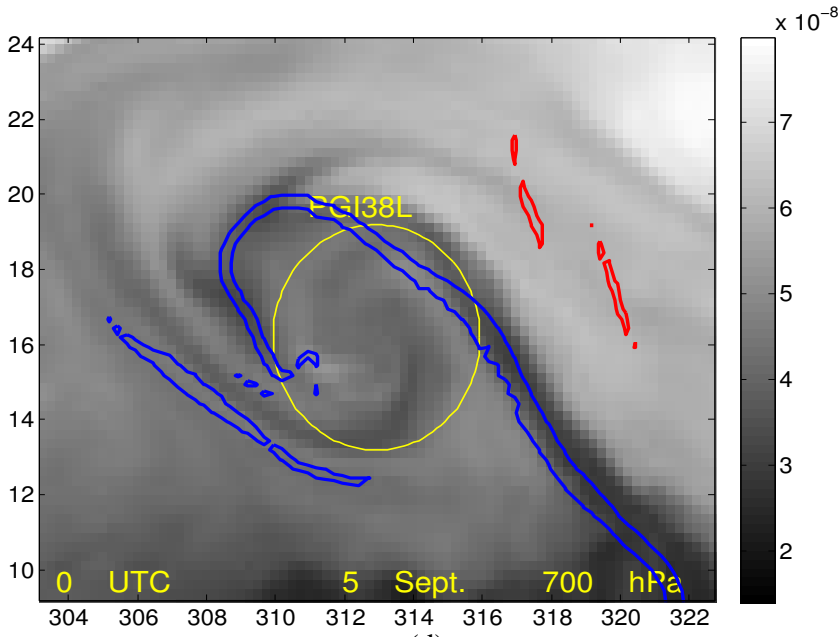

(d)

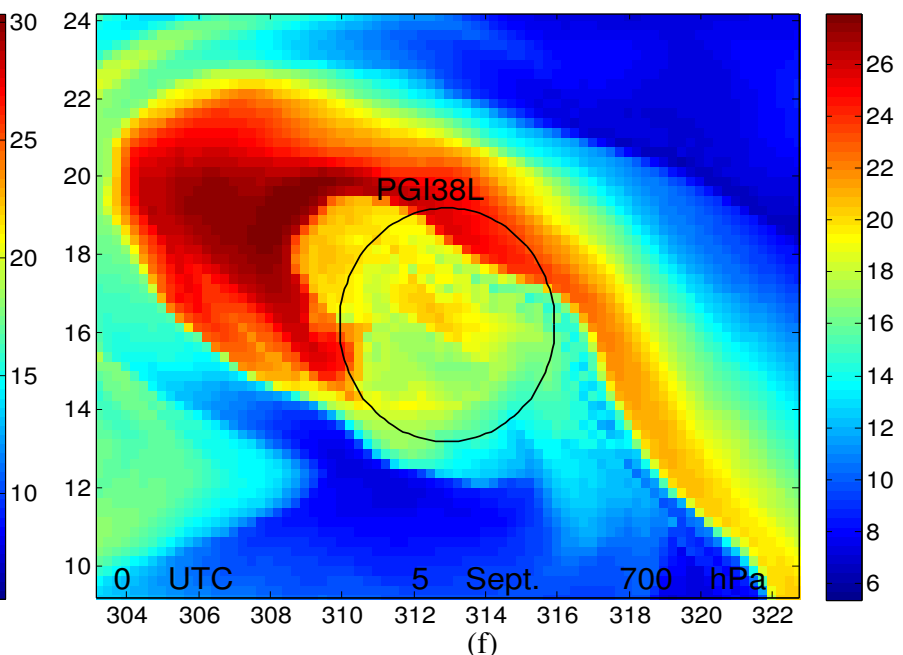

Fig. 8. Attracting LCSs (blue) and repelling LCSs (red) are represented as level contours of the FTLE field at $80 \%$ of the maximal FTLE values and overlaid on the relative humidity tracer field at $700 \mathrm{hPa}(\mathbf{a}, \mathbf{b})$ and the ozone mass fraction $\left(\mathrm{kg} \mathrm{kg}^{-1}\right)(\mathbf{c}, \mathbf{d})$ for ex-Gaston on 3 September (a, c) and 5 September (b, d). Backward trajectory lengths are shown for 3 and 5 September in (e) and (f). 


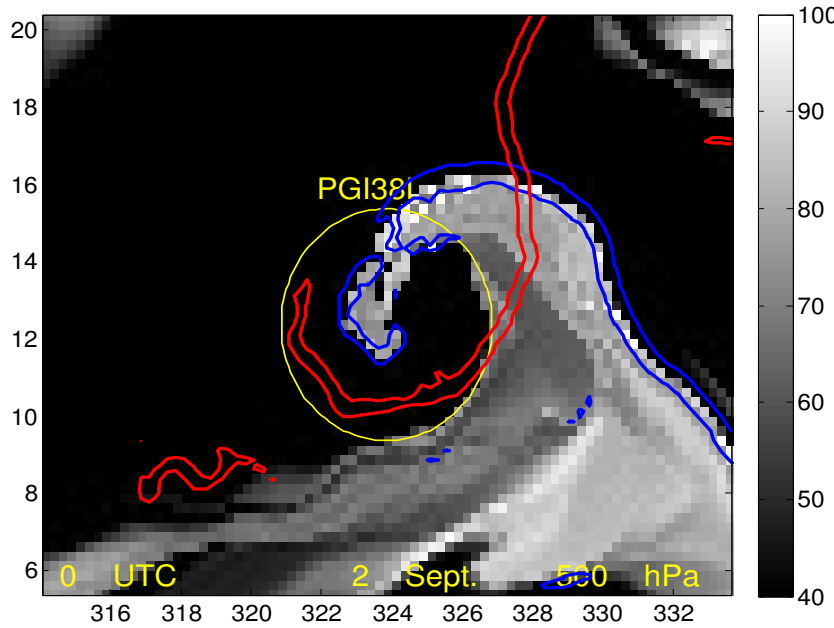

(a)

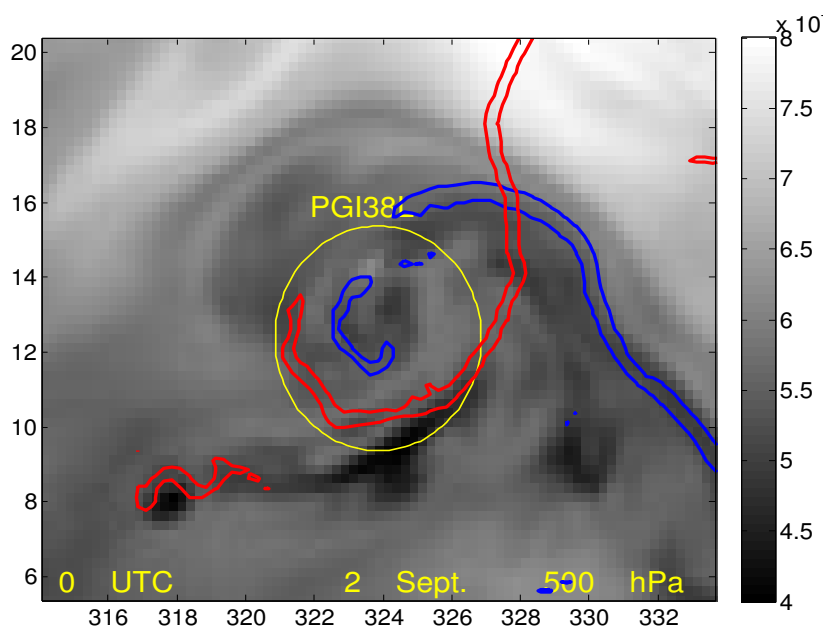

(c)

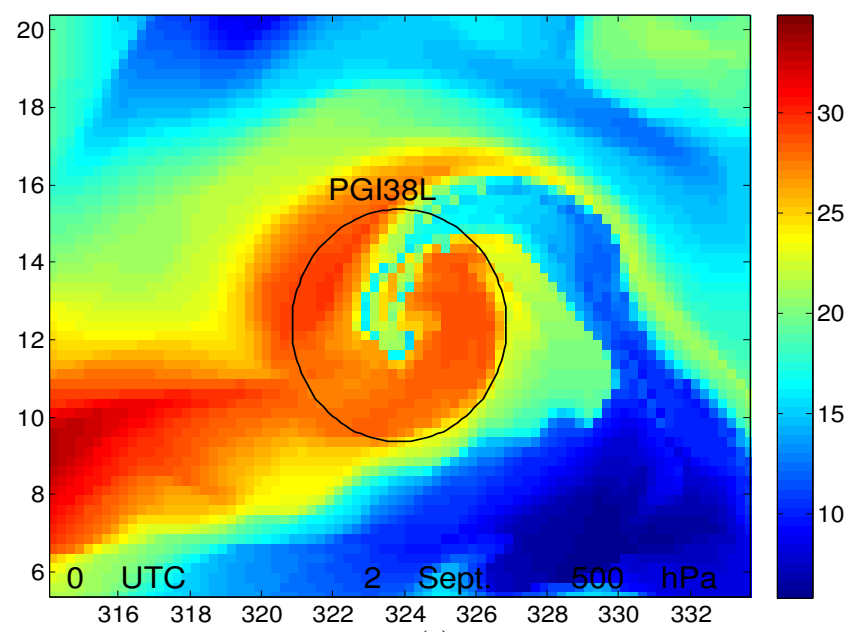

(e)

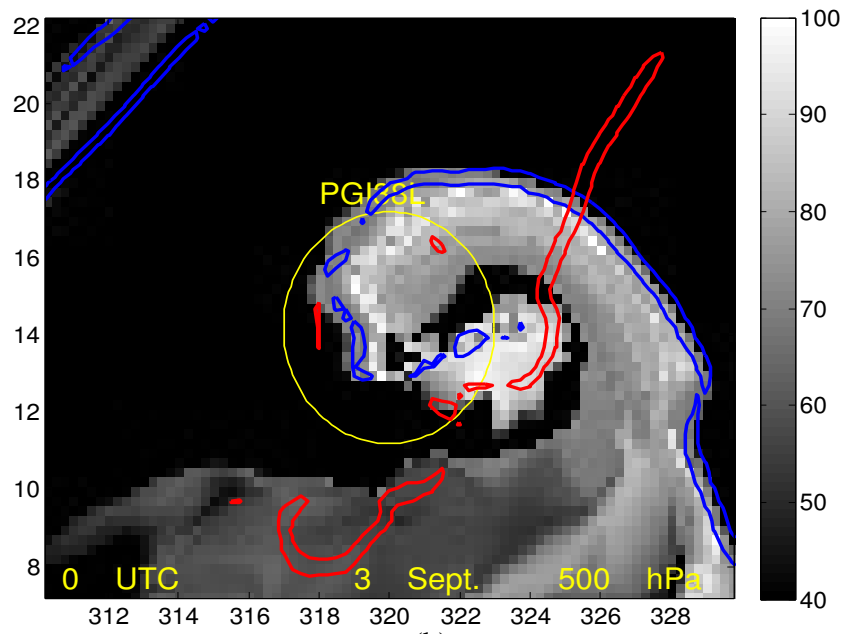

(b)

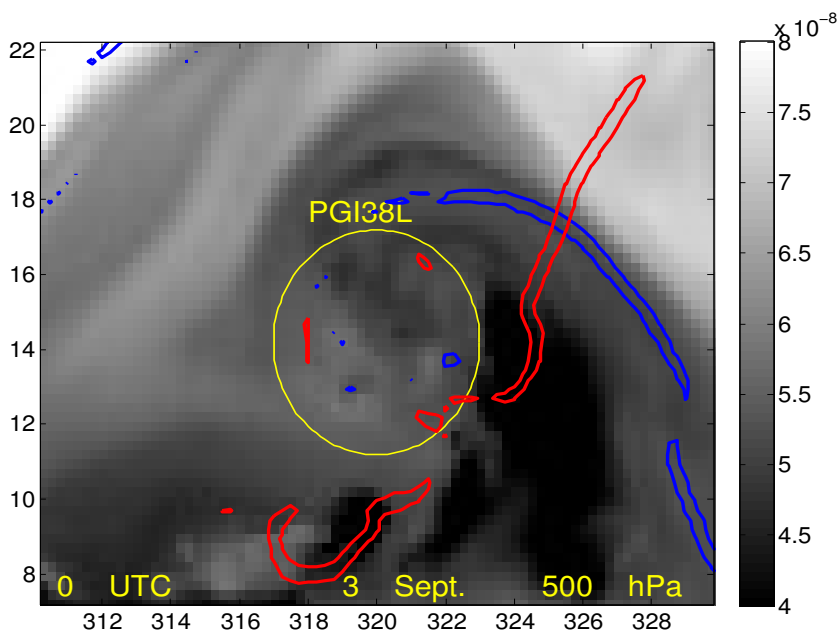

(d)

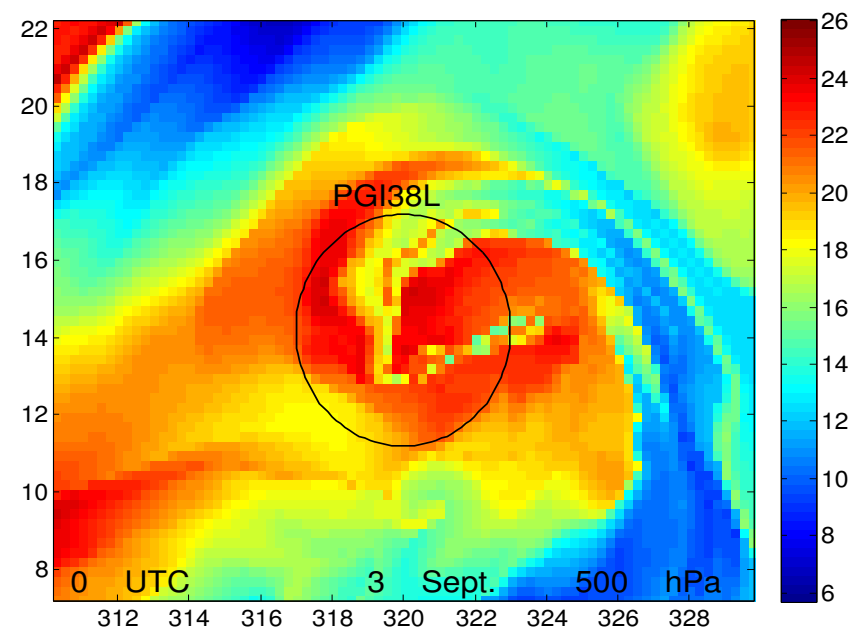

(f)

Fig. 9. Attracting LCSs (blue) and repelling LCSs (red) are represented as level contours of the FTLE field at $80 \%$ of the maximal FTLE values and overlaid on the relative humidity tracer field at $500 \mathrm{hPa}(\mathbf{a}, \mathbf{b})$ and the ozone mass fraction $\left(\mathrm{kg} \mathrm{kg}^{-1}\right)(\mathbf{c}, \mathbf{d})$ for ex-Gaston on 2 September $(\mathbf{a}, \mathbf{c})$ and 3 September $(\mathbf{b}, \mathbf{d})$. Trajectory lengths are shown for 2 September and 3 September in (e) and (f). 


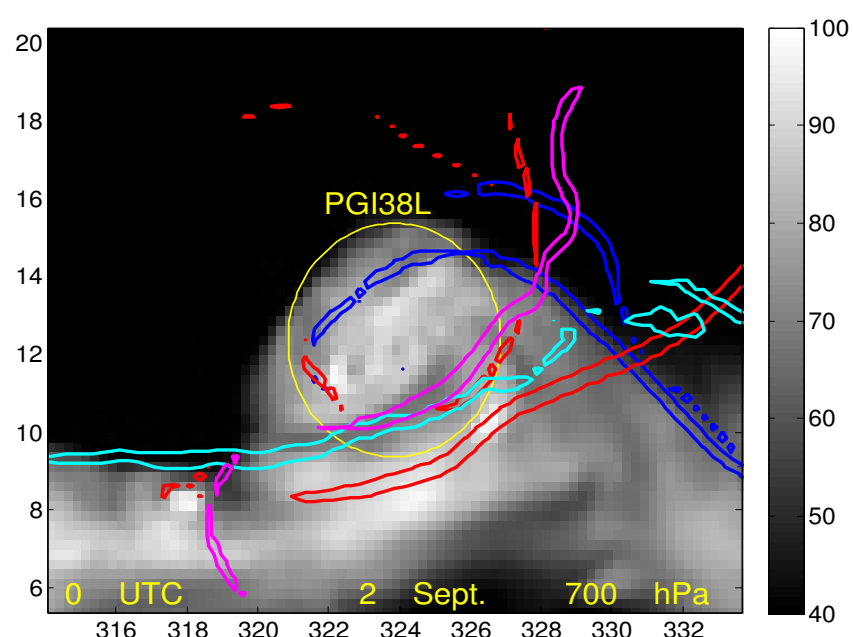

(a)

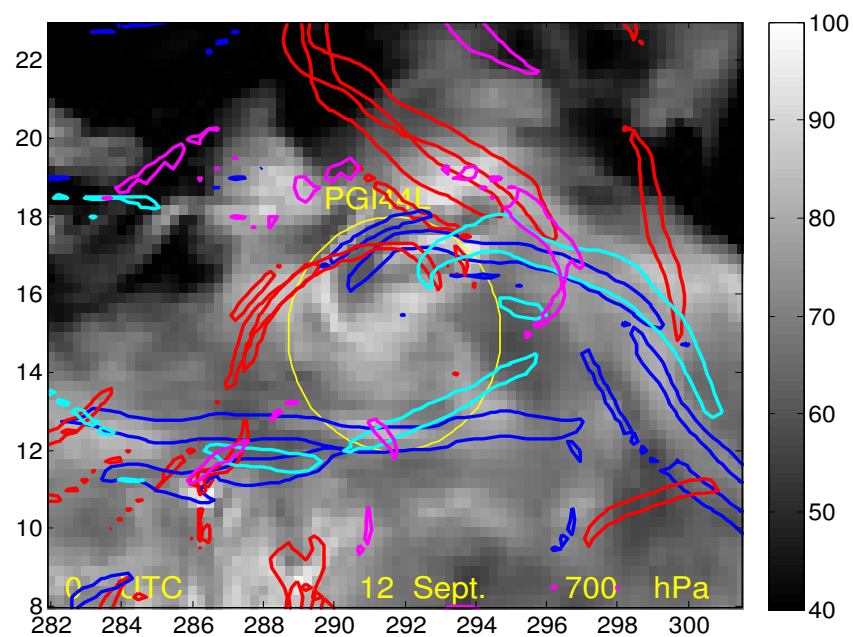

(c)

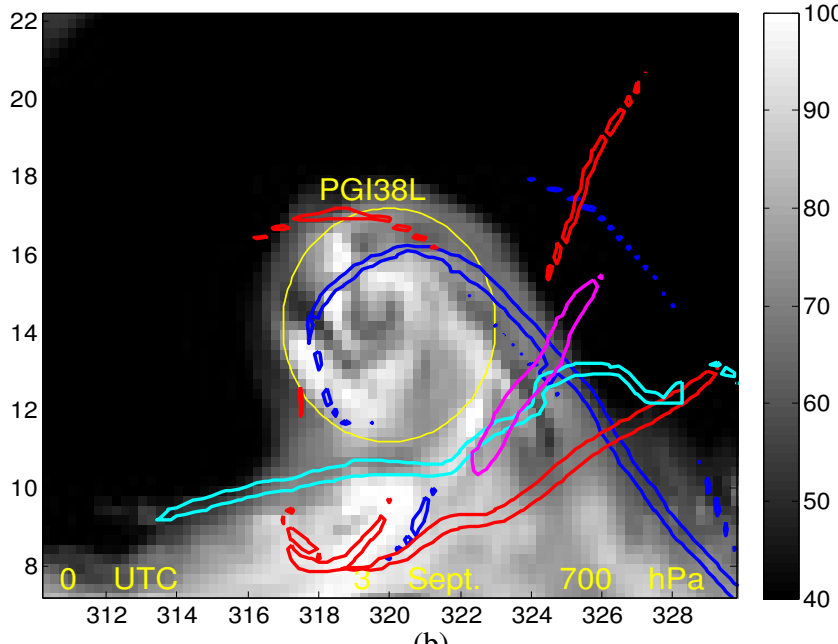

(b)

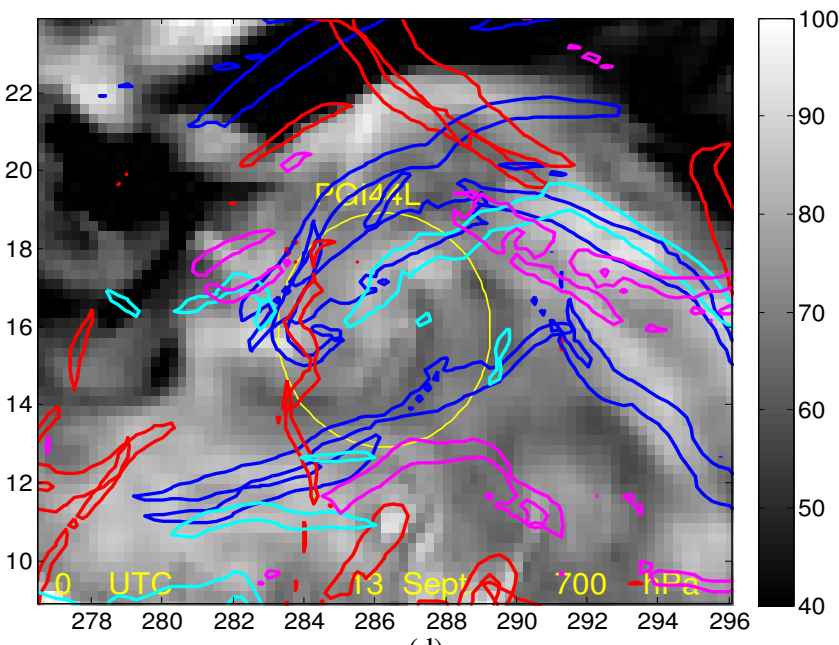

(d)

Fig. 10. Attracting LCSs (blue at 700 and $500 \mathrm{hPa}$, cyan at $925 \mathrm{hPa}$ ) and repelling LCSs (red at 500 and $700 \mathrm{hPa}$, magenta at $925 \mathrm{hPa}$ ) are represented as level contours of the FTLE field at $80 \%$ of the maximal FTLE values and overlaid on the $700 \mathrm{hPa}$ relative humidity (\%) field for ex-Gaston on 2 and 3 September in (a) and (b), and for pre Karl on 12 and 13 September in (c) and (d).

over the four day period of time before exhibiting a persistent region of central dense overcast on the day of development of an organized circulation center, 14 September (Montgomery et al. 2011). Karl intensified to a strong tropical storm on 15 September, and into a hurricane on 16 September, becoming a category 3 storm on 17 September, shortly before landfall in Veracruz, Mexico. The progressive increase of circulation is shown in the time series of area-averaged vorticity within the pouch region at various vertical levels in Fig. 4.

\subsubsection{Lagrangian analysis}

An analysis of the LCSs and their relation to moisture fields show that pre-Karl was far more favourable for development due to greater vertical alignment of the LCSs, and a more favourable location of LCSs relative to the pouch center. The absence of a repelling LCS enclosing the pouch center is also important since there is no pathway for particles to escape the pouch. The relative humidity tracer fields show that the particles entering the pouch are more moist than those entering Gaston.

The environment of pre-Karl is very moist at $925 \mathrm{hPa}$ around the pouch center (not shown), so that any entry of environmental air into the pouch will not reduce the overall moisture. At $700 \mathrm{hPa}$, Karl is still very moist, Fig. 11, but shows some dry air to the north and west of the pouch. The relative humidity structure of pre-Karl at $500 \mathrm{hPa}$ on 12 September, Fig. 12a is very similar to ex-Gaston on 1 September, Fig. 7a.

\subsubsection{LCSs at $700 \mathrm{hPa}$}

Figure 11a and $\mathrm{b}$ shows a repelling LCS (red) that appears to the north of the pouch on 12 September, and is intersected 
by an attracting LCS on 13 September. On 12 September, the LCSs form an enclosure of a small region in the pouch center. The attracting LCS remains to the north of the pouch and forms a teardrop shaped boundary around the pouch by 14 September. Evidence of strong cyclonic rotation can be seen on 15 September as both attracting and repelling LCSs wrap around the circulation center, Fig. 11.

The orientation of the LCS is almost $90^{\circ}$ different than for ex-Gaston, and the difference can most easily be seen by the location of the intersection of LCSs, which is located to the northeast of the pouch for Karl, Fig. 10b-d, instead of to the southeast for ex-Gaston, Fig. 7a-d. In addition, the pouch boundary of pre-Karl is further from the pouch center and requires a longer path for dry air to reach the pouch center. The longer pathway can be seen on 13 to 14 September, Fig. 11b$\mathrm{c}$ as a dry air region has wrapped from the north around the west side of the storm, and begins to enter from the southwest. The configuration of the LCSs for pre-Karl still allows moist air from the southeast to be entrained.

\section{LCSs at $500 \mathrm{hPa}$}

Figure 12 shows the LCS structure at $500 \mathrm{hPa}$ which is similar to $700 \mathrm{hPa}$, with an attracting LCS to the north of the pouch center. By 13 September, a repelling LCS intersects the attracting LCS at a similar location as the intersection at $700 \mathrm{hPa}$. Since the LCSs align vertically, they represent a two-dimensional boundary within the three-dimensional flow field. The location of the attracting LCS to the north of Karl is further north of the pouch center than for ex-Gaston, Fig. 12. The LCS boundary is not forced southward toward the pouch center by vertical wind shear, so the boundary maintains vertical alignment.

The LCSs protect Karl from importing air from the north with relative humidity levels of below $60 \%$ from 10 to 12 September. On 13 September, Figs. 11b and 12b, the LCSs at both $500 \mathrm{hPa}$ and $700 \mathrm{hPa}$ formed a boundary to dryer air wrapping from the north side of the pouch and entering from the west. The attracting LCS of the eastern hyperbolic trajectory differs from the similar LCS seen in Gaston in that it does not split and unzip. In addition, the repelling LCS of the eastern hyperbolic trajectory did not isolate the southern region of high moisture. Thus, the ITCZ remains intact as a potential source of fuel.

A complete enclosure around the pouch, surrounding most of the 3 degree radius circle around the pouch center can be seen on 15 September, Figs. 11d and 12d, though a small intrusion is later entrained at both 500 and $700 \mathrm{hPa}$ through lobe dynamics. This intrusion can also be seen in the ozone field, Fig. 13, but this entrainment is much slower than the open pathway that appeared for ex-Gaston, and did not occur until after genesis.

\section{Trajectory analysis}

The relative humidity tracer fields at $700 \mathrm{hPa}$ and $500 \mathrm{hPa}$ demonstrate that the air entering the pouch center of Karl was moist at both levels, Figs. 13 and 14 (left column), while dry air was blocked by the northern pouch boundary. The location of the boundary to the north of the pouch shows that moist air is drawn northward, which can also be seen by the increase in the size of the region of low ozone air, Figs. 13 and 14 (right column).

The length of trajectory paths is very different for Karl than for Gaston. While the trajectories for Gaston traveled the longest distances outside the pouch center, the trajectories for Karl that recirculated in the pouch center had the longest paths, Figs. 11c, d and 12c, d.

Figure 15 shows the final locations of backward trajectories originating in a 3 degree circle around the pouch center. While many particles originate outside the pouch boundary, they come from regions of high relative humidity. In contrast to Gaston, the trajectories approximately follow the Eulerian streamlines, though there are still particles that cross Eulerian streamlines.

The pouch averaged Lagrangian values are similar on all vertical levels, Fig. 5a, c. This property is in contrast to a disruption at $700 \mathrm{hPa}$ and above for ex-Gaston that causes lower averaged LCS values. The average Lagrangian values increase rapidly as pre-Karl begins to develop on 15 September, as the LCSs form the boundaries of closed circulation.

\section{Vertical structure}

The vertical alignment of LCSs is very different for preKarl and ex-Gaston. Figure 10 shows the vertical structure of the LCSs for pre-Karl at 500, 700 and $925 \mathrm{hPa}$ in b and d. The attracting (cyan) LCSs at $925 \mathrm{hPa}$ are already aligned with the attracting LCSs at 700 and $500 \mathrm{hPa}$ (blue) on 12 September and maintain their alignment until pre-Karl develops. In contrast, the vertical structure of the LCSs of exGaston, Fig. 10a, c, showed low-level LCSs perpendicular to the LCSs at $700 \mathrm{hPa}$ and above. We note that the vertical alignment of LCSs does not necessarily require the vertical alignment of Eulerian manifolds.

\section{Conclusions}

In this study, we have introduced finite-time Lagrangian methods for time-dependent flows to the study of tropical cyclogenesis. These methods were applied to developing tropical disturbances located along the wave trough axis of Easterly waves. The cat's eye boundary of the circulation was shown to be a robust feature even in the time-dependent context of the real tropical atmosphere. However, the timedependent boundaries of the cat's eye, which control transport in and out of the cat's eye, appear very different in the Lagrangian fields than they do in the Eulerian fields. 


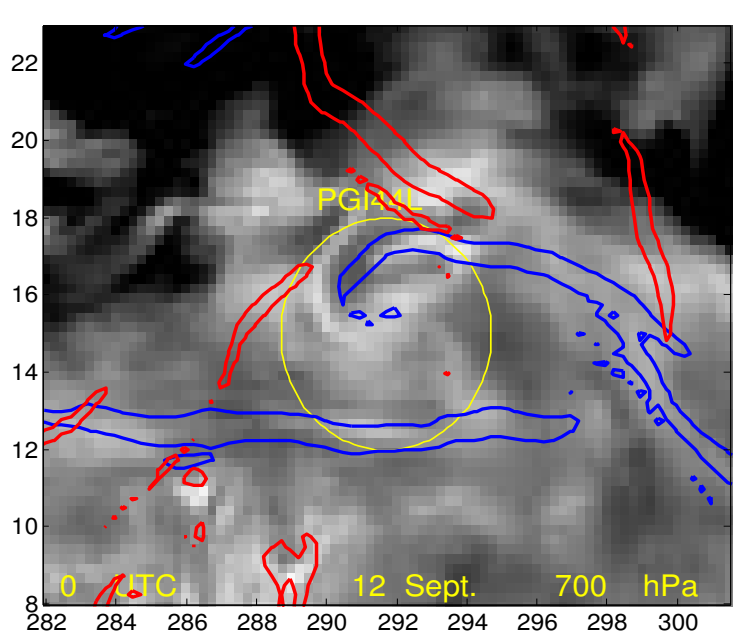

(a)

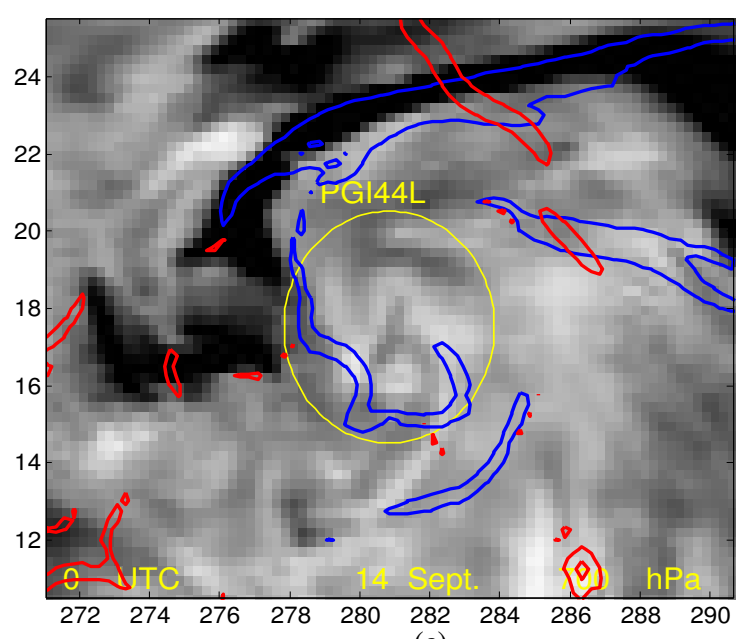

(c)

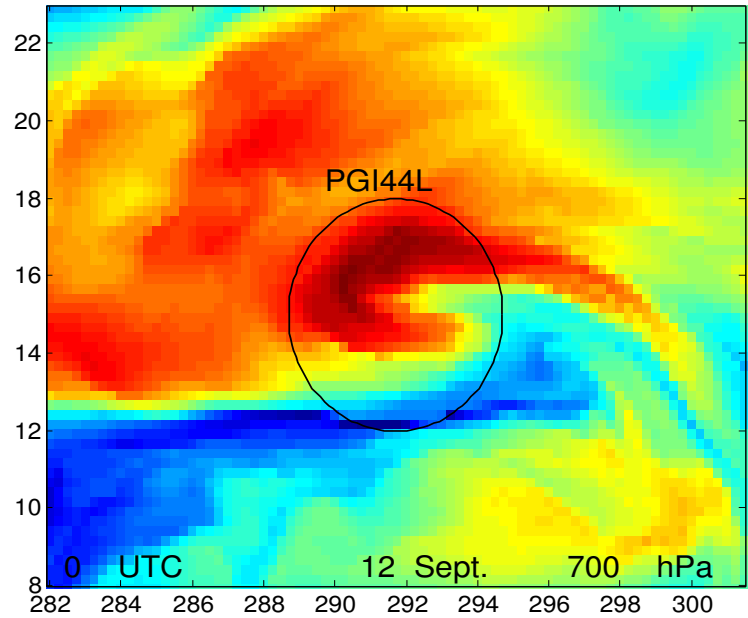

(e)
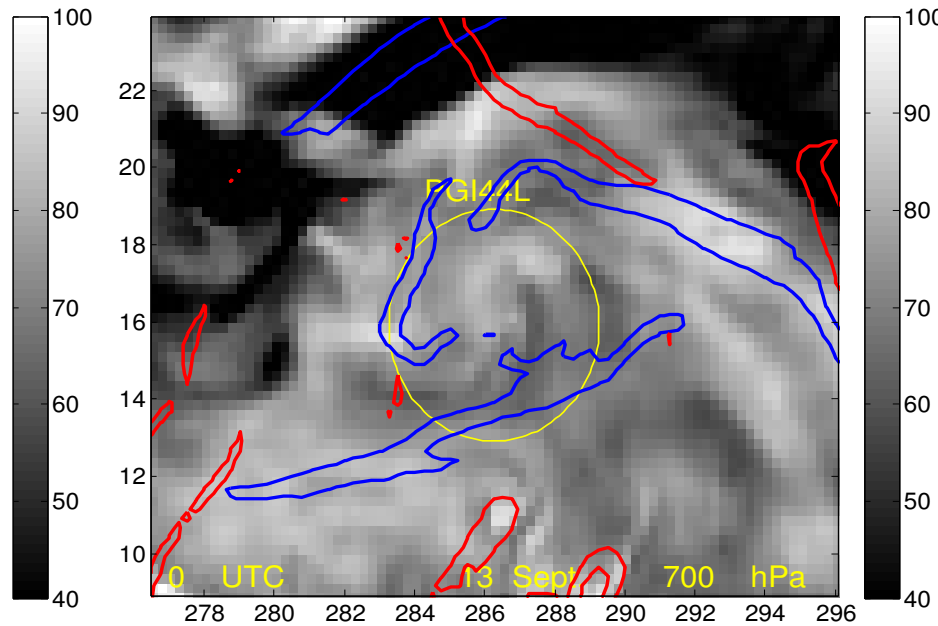

(b)
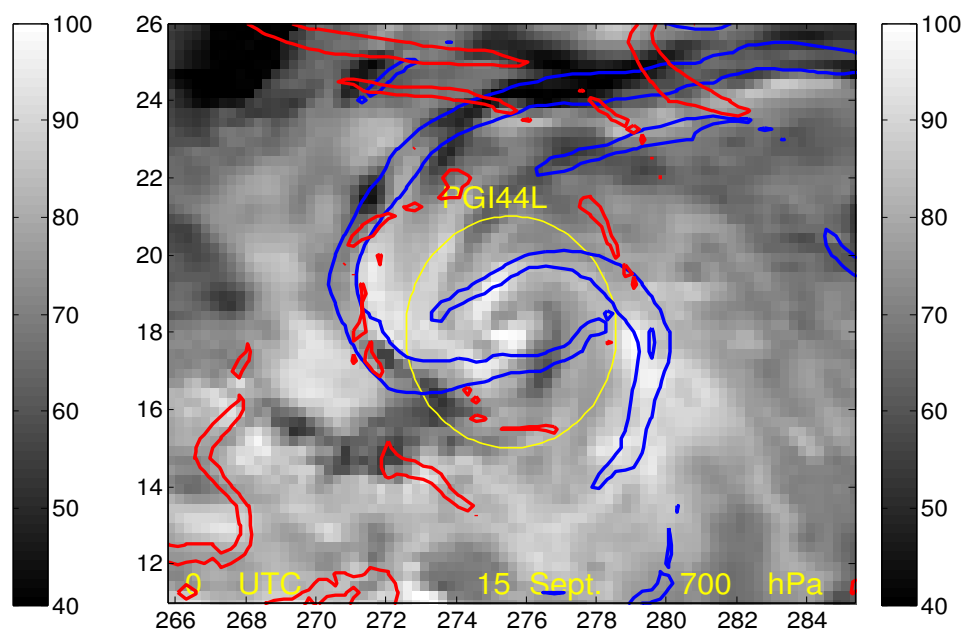

(d)
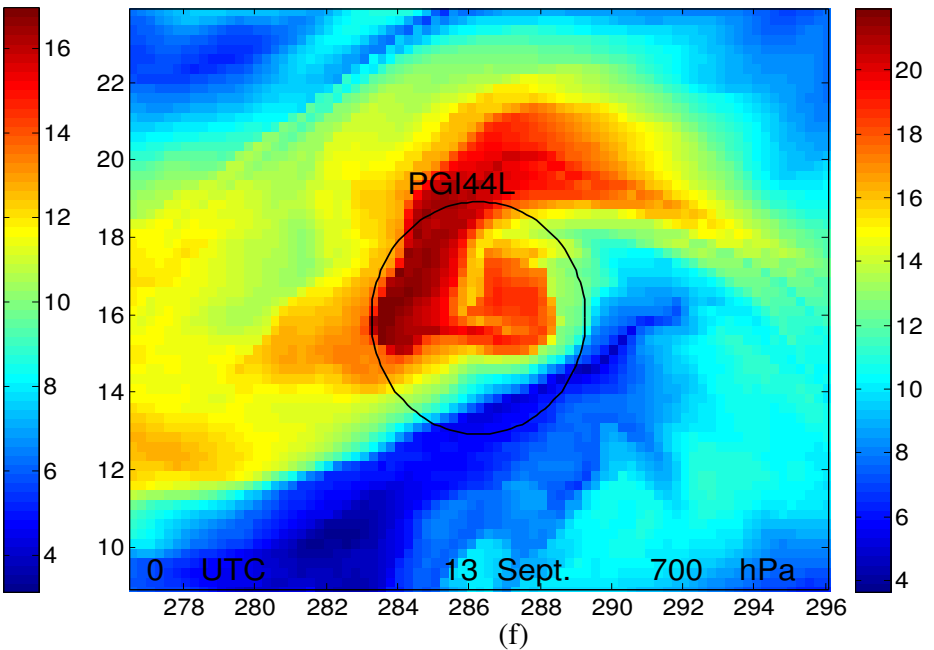

Fig. 11. Attracting LCSs (blue) and repelling LCSs (red) are represented as level contours of the FTLE field at $80 \%$ of the maximal FTLE values and overlaid on the relative humidity (\%) fields for pre-Karl at $700 \mathrm{hPa}$ from 12 September to 15 September (a-d). The backward trajectory lengths are shown on 12 September and 13 in (e) and (f). 


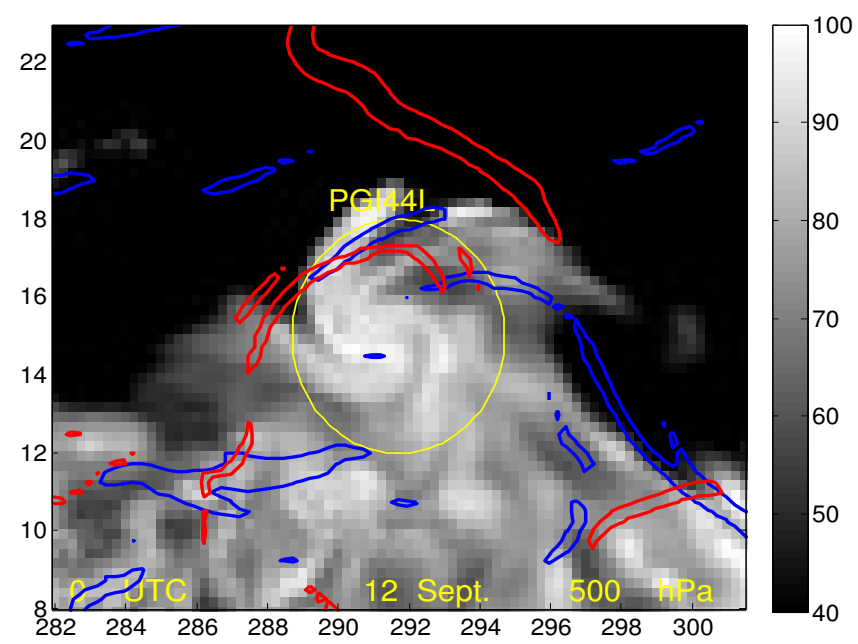

(a)

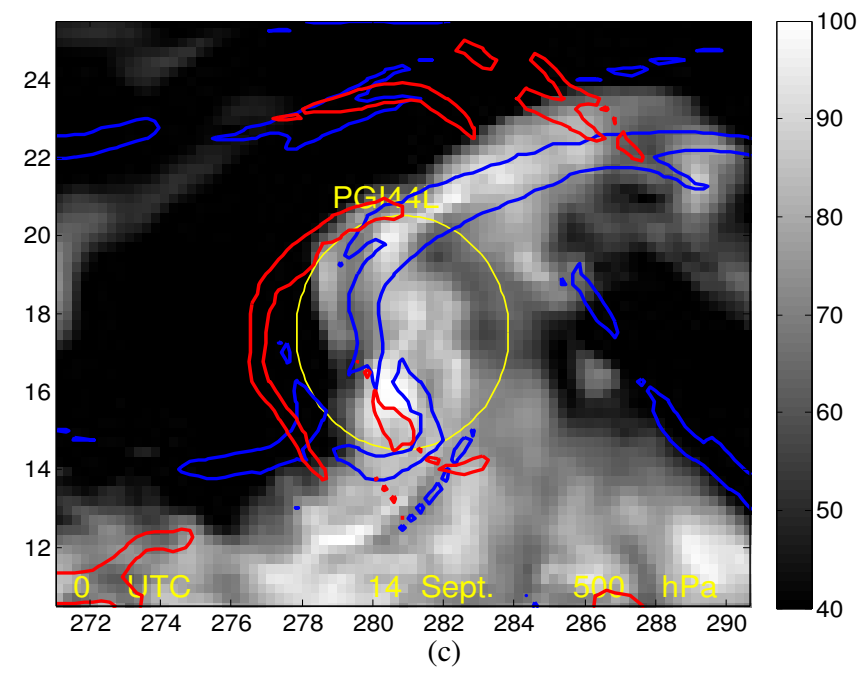

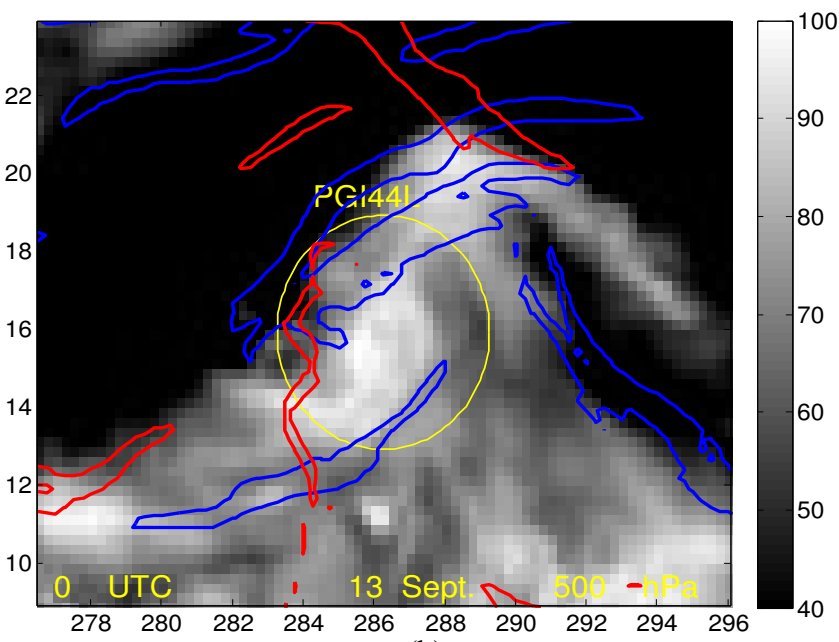

(b)

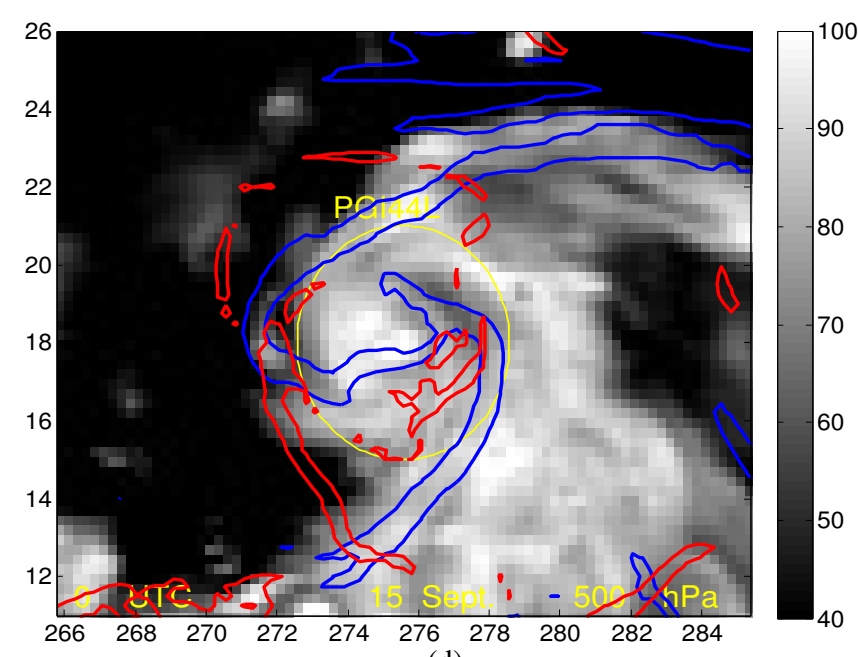

(d)

Fig. 12. Attracting LCSs (blue) and repelling LCSs (red) are represented as level contours of the FTLE field at $80 \%$ of the maximal FTLE values and overlaid on the relative humidity (\%) fields for pre-Karl at $500 \mathrm{hPa}$ from 12 to 15 September.

Though the eastern hyperbolic stagnation point and manifolds of the co-moving cat's eye could be associated with persistent LCSs, the western hyperbolic stagnation point did not provide persistent LCSs. When the LCSs from the western stagnation point existed, a boundary was formed where transport into the pouch was controlled by lobe dynamics. However, the absence of LCSs and existence of only a single hyperbolic trajectory changed the Lagrangian flow configuration, which allowed a direct pathway for dry air to enter the circulation.

In this setting, the Lagrangian reference frame has advantages over both Eulerian and co-moving frames. While the co-moving frame following the tropical disturbance showed closed streamlines, the Eulerian reference frame did not. However, streamlines from the co-moving frame were crossed by trajectories, as expected. In contrast, the Lagrangian boundaries are based on particle trajectories, per- sistent through the time-variation, and are impermeable to particle trajectories.

Attracting and repelling LCSs from FTLE fields were found in both a non-developing and developing disturbance to be the boundaries at the edge of the pouch associated with the cat's eye. The alignment and location of the LCSs defined pathways that determined the interaction of air masses with the tropical disturbance.

\subsection{Application to a developing and non-developing disturbance}

The Lagrangian techniques were applied to a developing and non-developing disturbance observed during the PREDICT experiment. We have specifically examined the cases of exGaston, which did not redevelop, and pre-Karl, which did develop into a hurricane. The LCSs showed boundaries which constrained the transport of moisture and vorticity. There was 


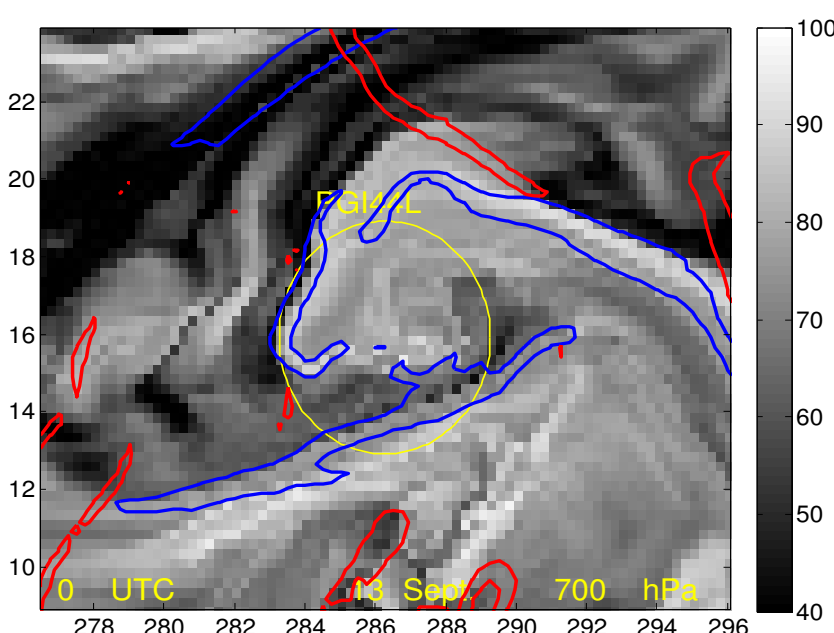

(a)

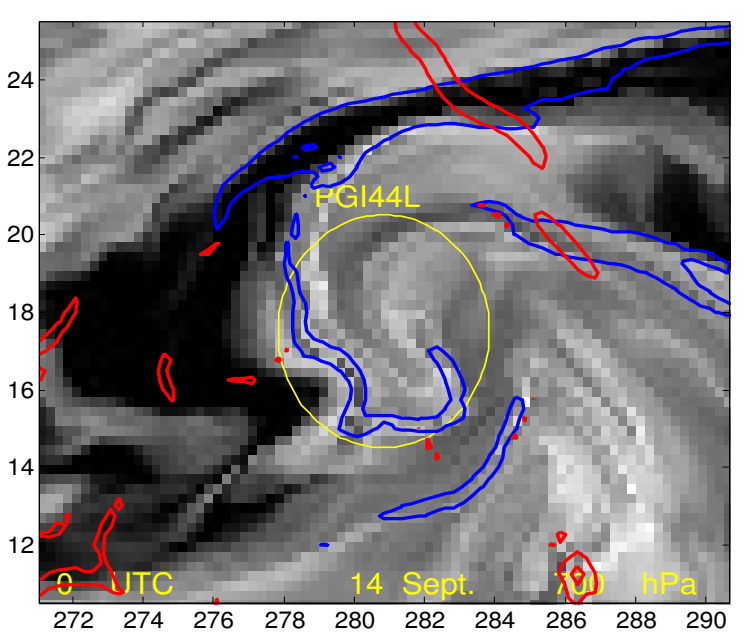

(c)

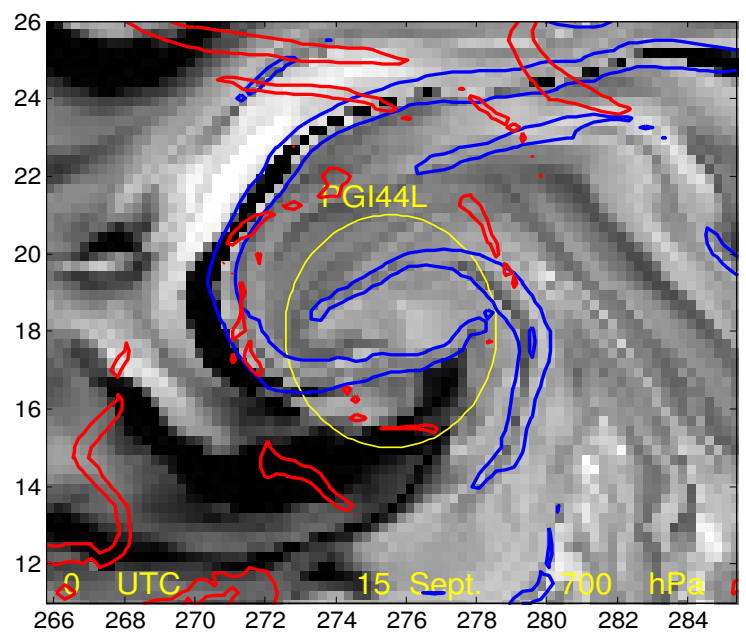

(e)
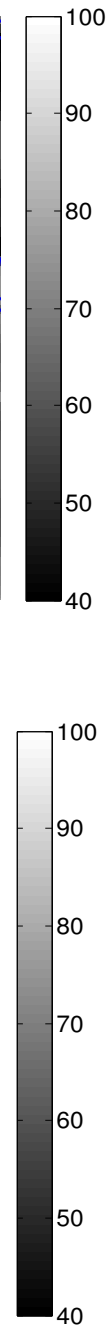

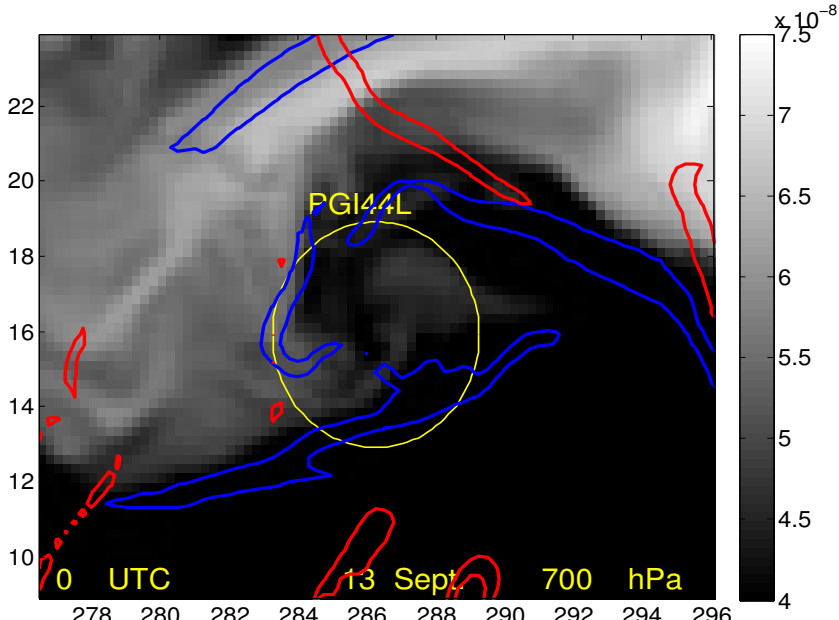

(b)

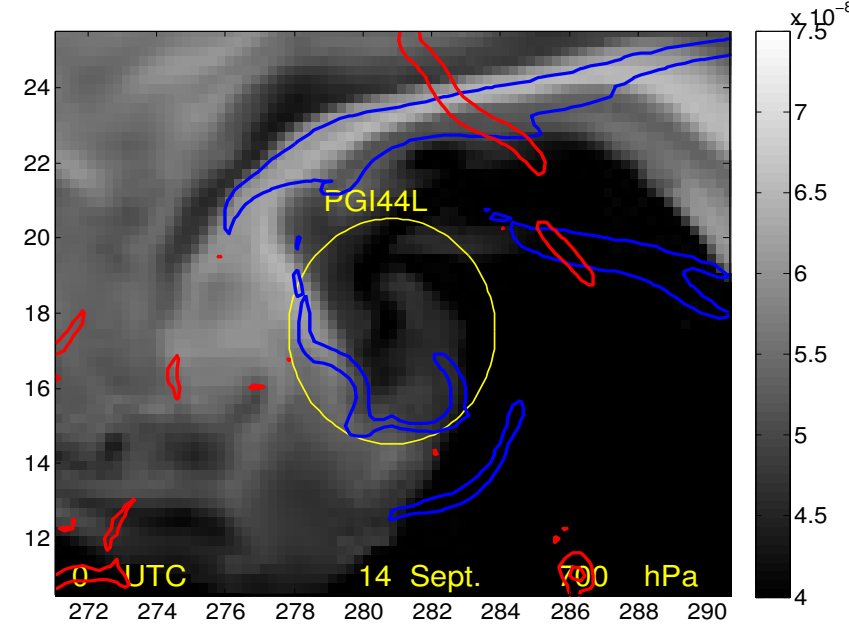

(d)

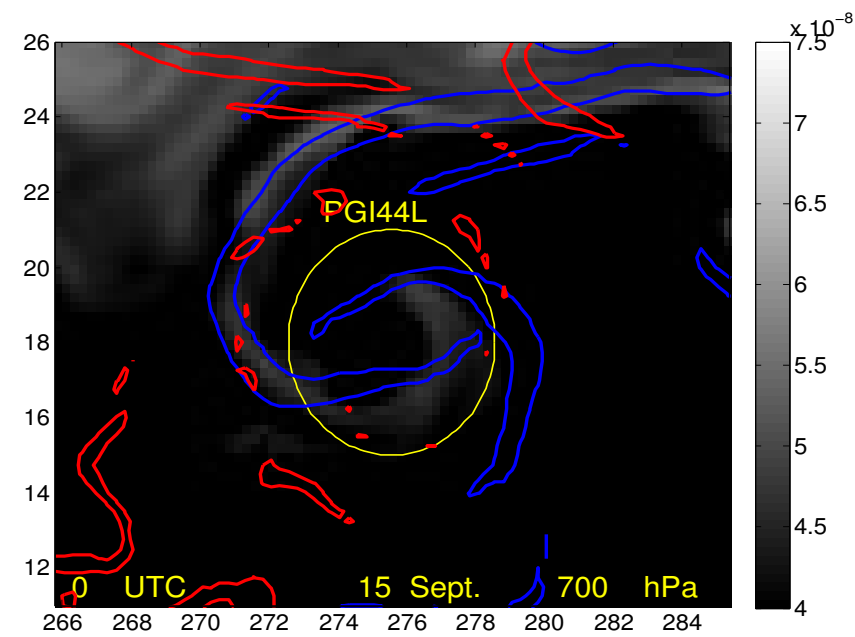

(f)

Fig. 13. Attracting LCSs (blue) and repelling LCSs (red) are represented as level contours of the FTLE field at $80 \%$ of the maximal FTLE values and overlaid on the RH tracer field at $700 \mathrm{hPa}$ for pre-Karl from 13 to 15 September (a, c, e). The LCSs are overlaid on the ozone mass fraction $\left(\mathrm{kg} \mathrm{kg}^{-1}\right)$ in (b), (d), and (f). 

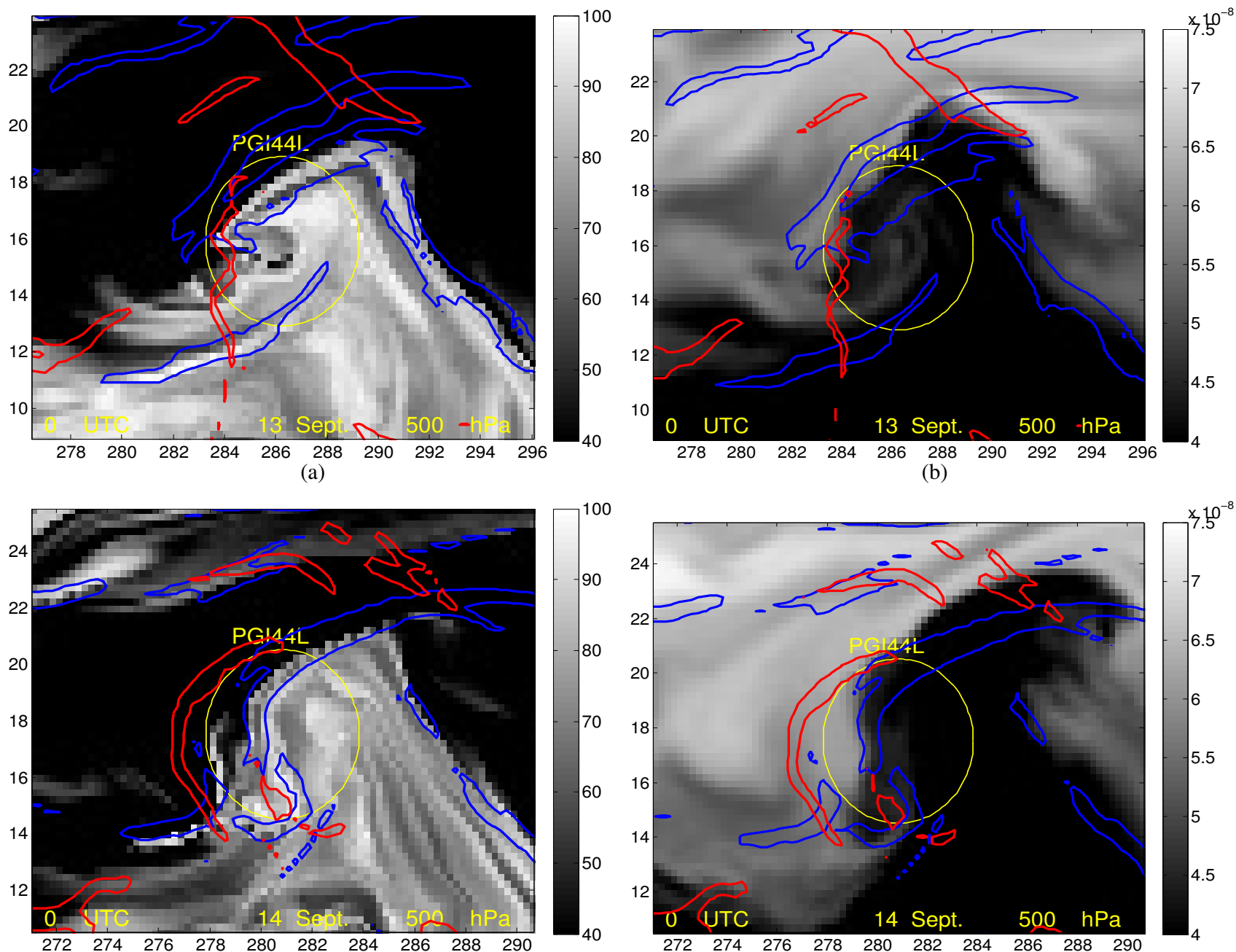

(c)

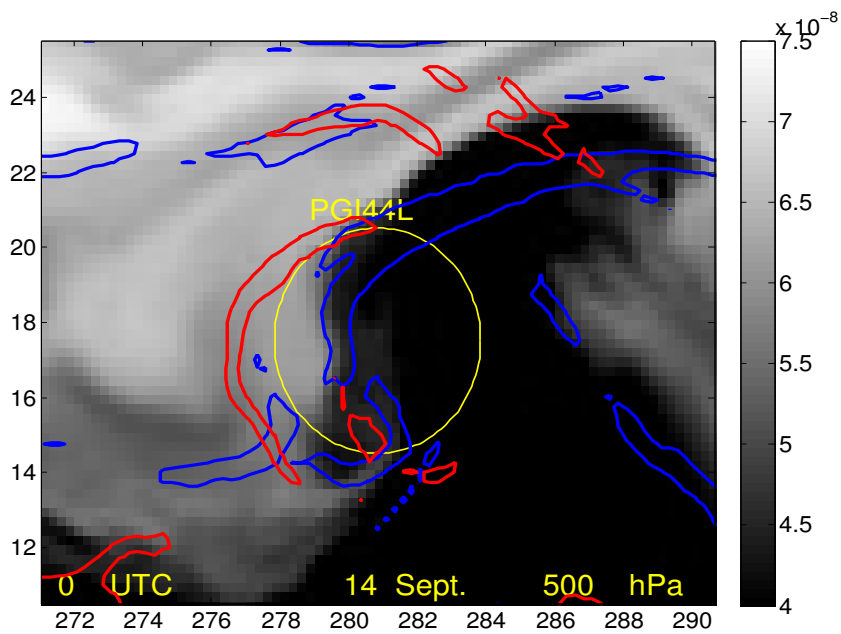

(d)

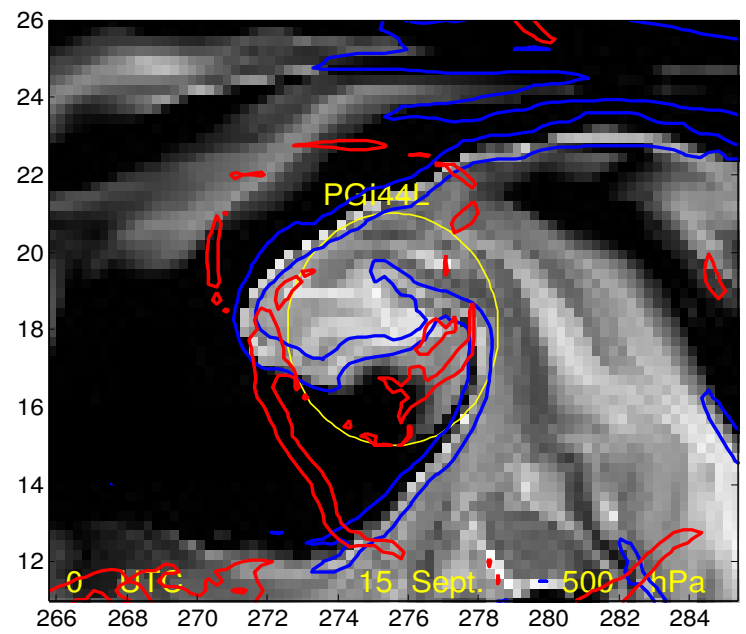

(e)
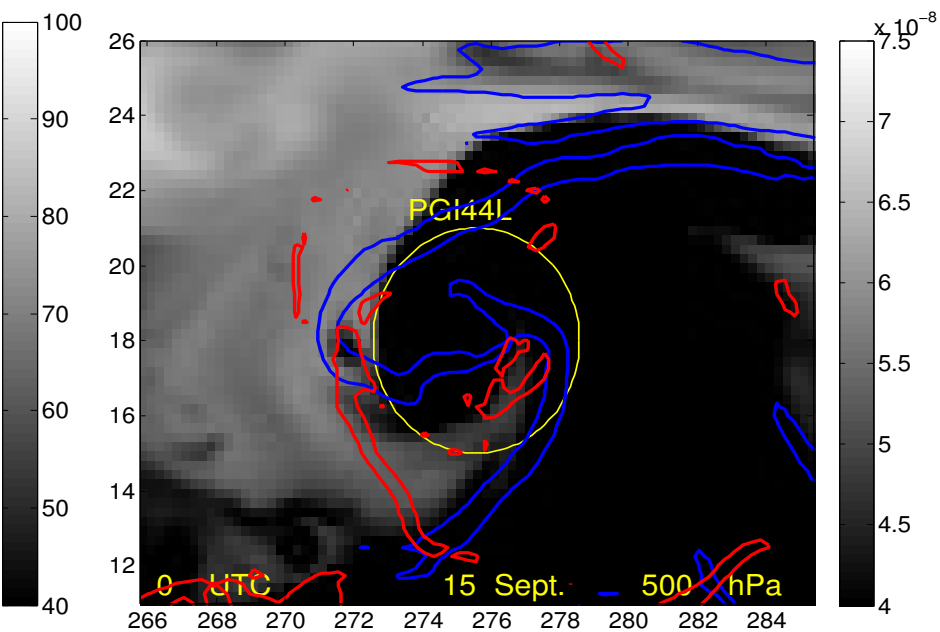

(f)

Fig. 14. Attracting LCSs (blue) and repelling LCSs (red) are represented as level contours of the FTLE field at $80 \%$ of the maximal FTLE values and overlaid on the RH tracer field at $500 \mathrm{hPa}$ for pre-Karl from 13 September to 15 September (a, c, e). The LCSs are overlaid on the ozone mass fraction $\left(\mathrm{kg} \mathrm{kg}^{-1}\right)$ in (b), (d), and (f). 


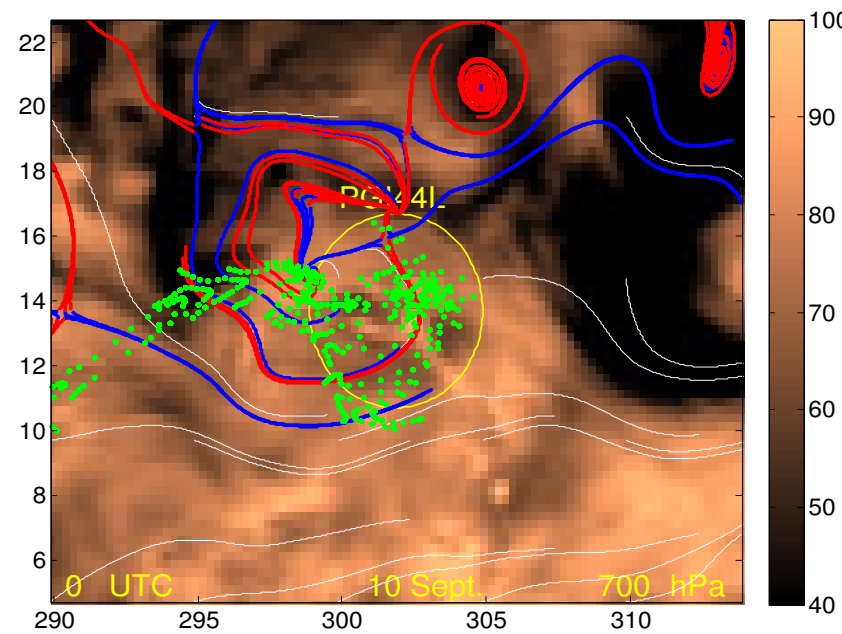

(a)



(c)

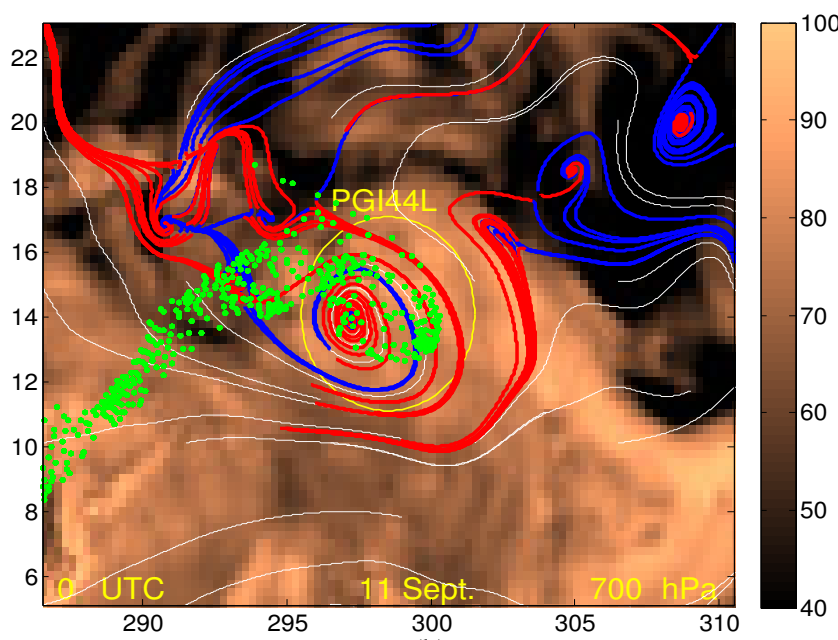

(b)

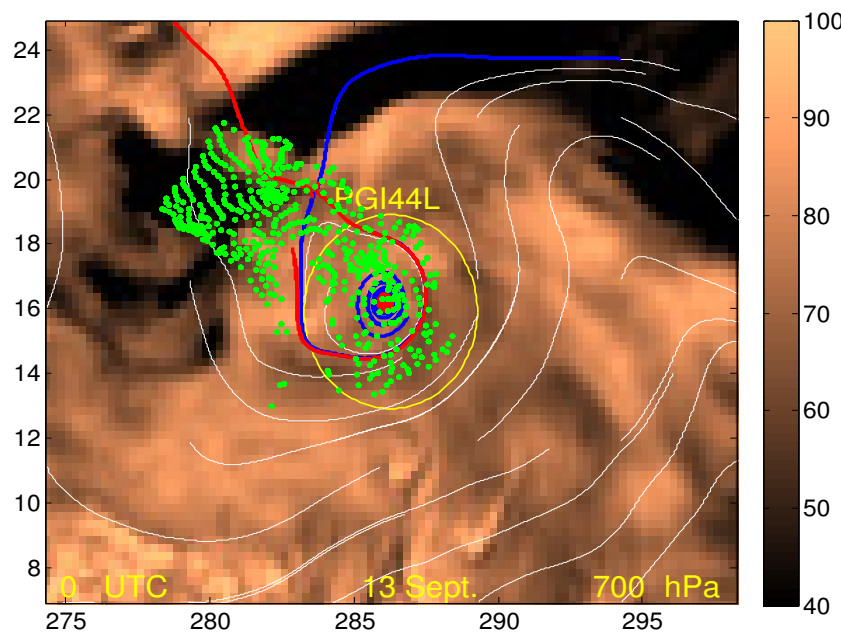

(d)

Fig. 15. The stable (red) and unstable (blue) manifolds of hyperbolic stagnation points and streamlines (white) of the co-moving frame are overlaid on the relative humidity (\%) field for Karl from 10 September to 13 September at $700 \mathrm{hPa}$. Green dots indicate the locations of particles that will be within 3 degrees of the pouch center in $48 \mathrm{~h}$.

a difference in LCSs and their position relative to moisture for the developing and non-developing cases.

Gaston and Karl had similar structure at lower levels of $850 \mathrm{hPa}$ and below but were far different at $700 \mathrm{hPa}$ and above. Gaston did not redevelop due to the intrusion of dry air, the expulsion of moist air with high vorticity, and a removal of a moisture source from the ITCZ. In addition, the presence of a repelling LCS in the inner core occurred as Gaston began to expel moist air. The LCSs showed the precise pathways and boundaries which were responsible for all of these processes. The vertical coherence of the pouch boundary is significantly degraded for ex-Gaston. The pouch boundary becomes tilted and the upper-level boundary becomes irreversibly detached due to impinging vertical shear.

The LCSs for pre-Karl were far more favourable for development. The LCSs blocked the intrusion of dry air, and retained a connection of moist air inflow from the ITCZ. A trajectory analysis showed the role of LCSs in organizing transport, and showed how Karl retained high moisture at all levels while Gaston did not.

\subsection{Further use of Lagrangian methods in cyclogenesis cases}

The computation of manifolds by first locating the hyperbolic trajectory is preferable for computing lobe dynamics. However, the lack of persistent stagnation points and ambiguity of proper reference frame make this computation difficult. Computing manifolds directly is difficult to implement in forecasting applications. Still, the approximations to Lagrangian manifolds through scalar field methods are present in both developing and non-developing disturbances, and the computation of FTLE fields in an automated manner makes the use of these methods applicable for 
forecasting purposes. These methods are particularly suitable for automation since they require no knowledge of pouch translation speed. Though Lagrangian methods are developed for three-dimensional flows, vertical velocity data for sparse temporal global model output would introduce a high degree of uncertainty to trajectory motions.

\subsection{Discussion and outlook}

LCSs show both the planar pathways for stirring, and reveal a complete picture of the vertical structure of the wave-pouch region. While the planar mixing in these cases is supported by an analysis of thermodynamics (Smith and Montgomery, 2012), further study of the three-dimensional kinematics and flow boundaries will help to explain the roles of stirring and downdrafts for importing dry air and expelling moist air. The methods used in this study will be applied to additional pregenesis cases from the 2010 PREDICT experiment to help identify the reasons for the development non-development of these pre-genesis cases. Real-time analysis using these methods will be explored also.

Acknowledgements. We gratefully acknowledge the support of NSF AGS-0733380 and NSF AGS-0851077, NOAA's Hurricane Research Division in Miami, FL, and NASA grants NNH09AK561 and NNG09HG031. The viewpoints presented in this paper are those of BR and MTM and do not necessarily represent the viewpoints of the government funding agencies. We wish to thank Gerald Thomsen and the German Weather Service, as well as Mark Boothe, for providing the ECMWF data.

Edited by: P. Haynes

\section{References}

Bell, M. M. and Montgomery, M. T.: Sheared deep vortical convection in pre-depression Hagupit during TCS08, Geophys. Res.Lett., 37, L06802, doi:10.1029/2009GL042313, 2010.

Branicki, M. and Wiggins, S.: Finite-time Lagrangian transport analysis: stable and unstable manifolds of hyperbolic trajectories and finite-time Lyapunov exponents, Nonlin. Processes Geophys., 17, 1-36, doi:10.5194/npg-17-1-2010, 2010.

Cohen, R. A. and Schultz, D. M.: Contraction rate and its relationship to frontogenesis, the Lyapunov exponent, fluid trapping, and airstream boundaries, Mon. Weather Rev., 133, 1353-1369, 2005.

Coulliette, C. and Wiggins, S.: Coulliette, C. and Wiggins, S.: Intergyre transport in a wind-driven, quasigeostrophic double gyre: An application of lobe dynamics, Nonlin. Processes Geophys., 7, 59-85, doi:10.5194/npg-7-59-2000, 2000.

Davis, C. A. and Ahijevych, D. A.: Mesoscale structural evolution of three tropical weather systems observed during PREDICT, J. Atmos. Sci., 69, 1284-1305, 2011.

d'Ovidio, F., Fernandez, V., and Hernandez-Garcia, E.: Mixing structures in the mediterranean sea from finite-size Lyapunov exponents. Geophy. Res. Lett., 31, L17203, doi:10.1029/2004GL020328, 2004.
d'Ovidio, F., Isern-Fontanet, J., Lopez, C., Hernandez-Garcia, E., and Garcia-Ladona, E.: Comparison between Eulerian diagnostics and finite-size Lyapunov exponents computed from altimetry in the algerian basin, Deep-Sea Res. I, 56, 15-31, 2009.

Duan, J. and Wiggins, S.: Fluid exchange across a meandering jet with quasiperiodic variability, J. Phys. Oceanogr., 26, 1176$1188,1996$.

Dunkerton, T. J., Montgomery, M. T., and Wang, Z.: Tropical cyclogenesis in a tropical wave critical layer: Easterly waves, Atmos. Chem. Phys., 9, 5587-5646, doi:10.5194/acp-9-5587-2009, 2009.

Evans, C., Archambault, H., Cordeira, J., Fritz, C., Galarneau Jr., T. J., Gjorgjievska, S., Griffin, A., Johnson, K., Komaromi, W., Monette, S., Muradyan, P., Murphy, B., Riemer, M., Sears, J., Stern, D., Tang, B., and Thompson, S.: The pre-depression investigation of cloud-sytems in the tropics (PREDICT) field campaign: Perspectives of early career scientists, B. Am. Meteor. Soc., 173-187, doi:10.1175/BAMS-D-11-00024.1, 2011.

Haller, G.: Finding finite-time invariant manifolds in twodimensional velocity fields, Chaos, 10, 99-108, 2000.

Haller, G.: Distinguished material surfaces and coherent structures in three-dimensional fluid flows, Phys. D, 149, 248-277, 2001.

Haller, G.: Lagrangian coherent structures from approximate velocity data. Phys. Fluids, 14:1851-1861, 2002.

Haller, G.: An objective view of a vortex. J. Fluid Mechanics, 525:1-26, 2005.

Haller, G.: A variational theory of hyperbolic Lagrangian Coherent Structures, Phys. D, 240, 574-598, 2011.

Haller, G. and Poje, A.: Finite time transport in aperiodic flows. Physica D, 119:352-380, 1997.

Haller, G. and Yuan, G.: Lagrangian coherent structures and mixing in two-dimensional turbulence, Phys. D, 147, 352-370, 2000.

Holton, J. R.: An Introduction to Dynamic Meteorology, edited by: Dmowska, R., Holton, J. R., and Rossby, H. T., Elsevier Academic Press, 535 pp., 2004.

Houze Jr., R. A., Lee, W. C., and Bell, M. M.: Convective contribution to the genesis of Hurricane Ophelia (2005). Mon. Weather Rev. 137, 2778-2800, 2009.

Hua, B. L. and Klein, P.: An exact criterion for the stirring properties of nearly two-dimensional turbulence, Phys. D, 113, 98-110, 1998.

Huber, M., McWilliams, J. C., and Ghil, M.: A climatology of turbulent dispersion in the troposphere, J. Atmos. Sci., 58, 2377 2394, 2001.

Ide, K., Small, D., and Wiggins, S.: Distinguished hyperbolic trajectories in time-dependent fluid flows: analytical and computational approach for velocity fields defined as data sets, Nonlin. Processes Geophys., 9, 237-263, doi:10.5194/npg-9-237-2002, 2002.

T.Y. Koh and B. Legras. Hyperbolic lines and the stratospheric polar vortex, Chaos, 2, 382-394, 2002.

Koh, T. Y. and Plumb, R. A.: Lobe dynamics applied to barotropic Rossby-wave breaking, Phys. Fluids, 12, 1518-1528, 2000.

Joseph, B. and Legras, B.: Relation between kinematic boundaries, stirring, and barriers for the Antarctic polar vortex, J. Atmos. Sci., 59, 1198-1212, 2002.

G. Kilroy and R. K. Smith A numerical study of rotating convection during tropical cyclogenesis, Q. J. Roy. Meteorol. Soc., doi:10.1002/qj.2022, 2012. 
Lapeyre, G., Klein, P., and Hua, L.: Does the tracer gradient vector align with the strain eigenvectors in 2D turbulence?, Phys. Fluids, 11, 3729-3737, 1999.

Lukovitch, J. and Sheperd, T.: Stirring and mixing in twodimensional divergent flow, J. Atmos. Sci., 62, 3933-3954, 2005.

Malhotra, N. and Wiggins, S.: Geometric structures, lobe dynamics, and Lagrangian transport in flows with aperiodic timedependence, with applications to Rossby wave flow, J. Nonlinear Sci., 8, 401-456, 1999.

Mancho, A. M., Small, D., Wiggins, S., and Ide, K.: Computation of stable and unstable manifolds of hyperbolic trajectories in two-dimensional, aperiodically time-dependent vector fields, Phys. D, 182, 188-222, 2003.

Mancho, A. M., Small, D., and Wiggins, S.: Computation of hyperbolic trajectories and their stable and unstable manifolds for oceanographic flows represented as data sets, Nonlin. Processes Geophys., 11, 17-33, doi:10.5194/npg-11-17-2004, 2004.

Montgomery, M. T., Davis, C., Dunkerton, T. J., Wang, Z., Velden, C., Torn, R., Majumdar, S. J., Zhang, F., Smith, R. K., Bosart, L., Bell, J. S., Haase, M. M., Heymsfield, A., Jensen, J., Campos, T., and Boothe, M. A.: The pre-depression investigation of cloud systems in the tropics (PREDICT) experiment: Scientific basis, new analysis tools and some first results, B. Am. Meteorol. Soc., 93, 153-172, 2012.

O'Farrell, C. and Dabiri, J. O.: A Lagrangian approach to identifying vortex pinch-off, Chaos, 20, 1-9, 2010.

Ottino, J. M.: The kinematics of mixing: stretching, chaos, and transport, Cambridge University Press, 364 pp., 1990.

Pierrehumbert, R. T.: Large scale horizontal mixing in planetary atmospheres, Phys. Fluids A, 3, 1250-1260, 1991.

Reasor, P. D., Montgomery, M. T., and Bosart, L.: Mesoscale observations of the genesis of Hurricane Dolly (1996), J. Atmos. Sci., 62, 3151-317, 2005.

Riemer, M. and Montgomery, M. T.: Simple kinematic models for the environmental interaction of tropical cyclones in vertical wind shear, Atmos. Chem. Phys., 11, 9395-9414, doi:10.5194/acp-11-9395-2011, 2011.
Rogerson, A. M., Miller, P. D., Pratt, J. L., and Jones, C. K. R. T.: Lagrangian motion and fluid exchange in a barotropic meandering jet, J. Phys. Oceanogr., 29, 2635-2655, 1999.

Rutherford, B. and Dangelmayr, G.: A 3D Lagrangian hurricane eye-eyewall computation, Q. J. Roy. Meteor. Soc., 136, 19311944, 2010.

Rutherford, B., Dangelmayr, G., and Montgomery, M. T.: Lagrangian coherent structures in tropical cyclone intensification, Atmos. Chem. Phys., 12, 5483-5507, doi:10.5194/acp-12-54832012, 2012.

Salman, H., Ide, K., and Jones, C. K. R. T.: Using flow geometry for drifter deployment in Lagrangian data assimilation, Tellus A., 60, 321-335, 2008.

Schubert, W. H., Montgomery, M. T., Taft, R. K., Guinn, T. A., Fulton, S. R., Kossin, J. P., and Edwards, J. P.: Polygonal eyewalls, asymmetric eye contraction, and potential vorticity mixing in hurricanes, J. Atmos. Sci., 56, 1197-1223, 1999.

Shadden, S.: A dynamical systems approach to unsteady flows. PhD thesis, California Institute of Technology, CA, USA, 2006.

Shadden, S. C., Lekien, F., and Marsden, J. E.: Definition and properties of Lagrangian coherent structures from finite-time Lyapunov exponents in two-dimensional aperiodic flows, Phys. D, 212, 271-304, 2005.

Smith, R. K. and Montgomery, M. T.: Observations of the convective environment in developing and non-developing tropical disturbances, Q. J. Roy. Meteor. Soc., 138, 1721-1739, 2012.

Tang, W., Mathur, M., Haller, G., Hahn, D., and Ruggiero, F.: Lagrangian coherent structures near a subtropical jet stream, J. Atmos. Sci., 67, 2307-2319, 2009.

Wang, Z.: Thermodynamic aspects of tropical cyclone formation, J. Atmos. Sci., 69, 2433-2451, doi:10.1175/JAS-D-11-0298.1, 2012.

Weiss, J. B. and Provenzale, A.: Transport and mixing in geophysical flows, Springer, New York, USA, 261 pp., 2008. 